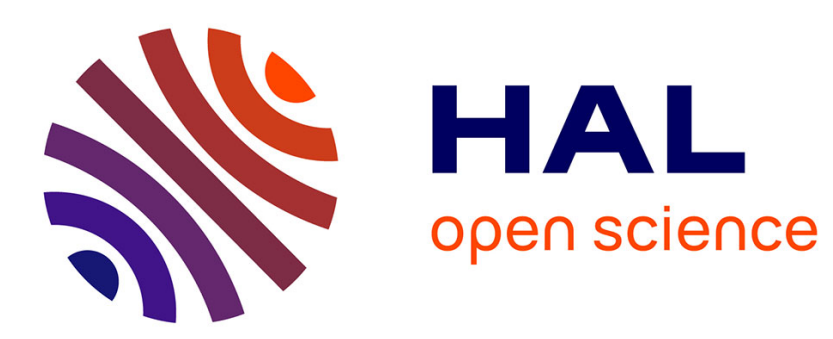

\title{
Yield criteria for shape memory materials : convexity conditions and surface transport
}

\author{
Mohamed Rachid Laydi, Christian Lexcellent
}

\section{To cite this version:}

Mohamed Rachid Laydi, Christian Lexcellent. Yield criteria for shape memory materials : convexity conditions and surface transport. Mathematics and Mechanics of Solids, 2010, 15 (2), pp.165 - 208. $10.1177 / 1081286508095324$. hal-02300127

\section{HAL Id: hal-02300127 \\ https://hal.science/hal-02300127}

Submitted on 29 Sep 2019

HAL is a multi-disciplinary open access archive for the deposit and dissemination of scientific research documents, whether they are published or not. The documents may come from teaching and research institutions in France or abroad, or from public or private research centers.
L'archive ouverte pluridisciplinaire HAL, est destinée au dépôt et à la diffusion de documents scientifiques de niveau recherche, publiés ou non, émanant des établissements d'enseignement et de recherche français ou étrangers, des laboratoires publics ou privés. 
archives-ouvertes

\title{
Yield criteria for shape memory materials : convexity conditions and surface transport
}

\author{
Mohamed Rachid Laydi, Christian Lexcellent
}

\section{To cite this version:}

Mohamed Rachid Laydi, Christian Lexcellent. Yield criteria for shape memory materials : convexity conditions and surface transport. Mathematics and Mechanics of Solids (MMS), 2010, 15 (2), pp.165 - 208. hal-02300127

\section{HAL Id: hal-02300127 \\ https://hal.archives-ouvertes.fr/hal-02300127}

Submitted on 29 Sep 2019

HAL is a multi-disciplinary open access archive for the deposit and dissemination of scientific research documents, whether they are published or not. The documents may come from teaching and research institutions in France or abroad, or from public or private research centers.
L'archive ouverte pluridisciplinaire HAL, est destinée au dépôt et à la diffusion de documents scientifiques de niveau recherche, publiés ou non, émanant des établissements d'enseignement et de recherche français ou étrangers, des laboratoires publics ou privés. 


\title{
Yield Criteria for Shape Memory Materials: \\ Convexity Conditions and Surface Transport
}

\author{
MOHAMED RACHID LAYDI \\ CHRISTIAN LEXCELLENT \\ Département de Mécanique Appliquée FEMTO-ST, 24 Rue de l'épitaphe, 25000 Besançon, \\ France
}

(Received 13 March 2008; accepted 13 June 2008)

\section{Dedicated to Professor Bogdan Raniecki on the occasion of his 70th birthday.}

\begin{abstract}
We study the yield conditions of phase transformation initiation for shape memory alloys exhibiting asymmetry between tension and compression. An extension of the choice of the classical invariant parameters such as those of Lode is proposed. A necessary and sufficient condition of convexity of these surfaces representing the elastic domain of austenite in the stress space, is established. Moreover the transport of these surfaces in the space of effective transformations strains of martensite is done. Hence, the duality between these two spaces is built. Some applications involving $\mathrm{Cu}-\mathrm{Al}-\mathrm{Be}$ and NiTi shape memory alloys end the purpose.
\end{abstract}

Key Words: Yield surface, shape memory alloy, convexity, duality, phase transformation

\section{INTRODUCTION}

As is the case in classical plasticity, the determination of the yield surfaces of phase transformation initiation is a key point for modeling shape memory alloys (SMA) behavior. The SMA exhibit an asymmetric behavior between tension and compression. Namely the yield stress is different between these two different uniaxial loadings [1]. Moreover, the SMA are considered as pressure-insensitive alloys. It means that the phase transformation between austenite and martensite is accompanied with no volume change. As pointed out by Raniecki and Lexcellent [2], the dependence of the yield criterion on the first invariant of stress tensor $\boldsymbol{\sigma}\left(I_{1} \equiv \operatorname{tr}(\boldsymbol{\sigma})\right)$ is negligible. Only the second and third invariants of the deviator of $\boldsymbol{\sigma}$ are involved. As for phase transformation initiation, in the tensor $\boldsymbol{\sigma}$, the material is only sensitive to the stress deviator $\mathbf{S}_{\sigma} \equiv \operatorname{dev}(\boldsymbol{\sigma})$.

In addition to these two classical Lode variables, i.e. the invariant and the angle, a third variable which is the greatest eigenvalue of reduced stress deviator $N_{\sigma}$ is introduced in order to simplify the calculations and to generalize the formulations. 


$$
\theta_{\sigma}=\operatorname{angle}\left(S_{\sigma} ; e_{1}\right) \quad \theta_{\varepsilon}=\operatorname{angle}\left(\varepsilon_{\sigma} ; e_{1}\right)
$$

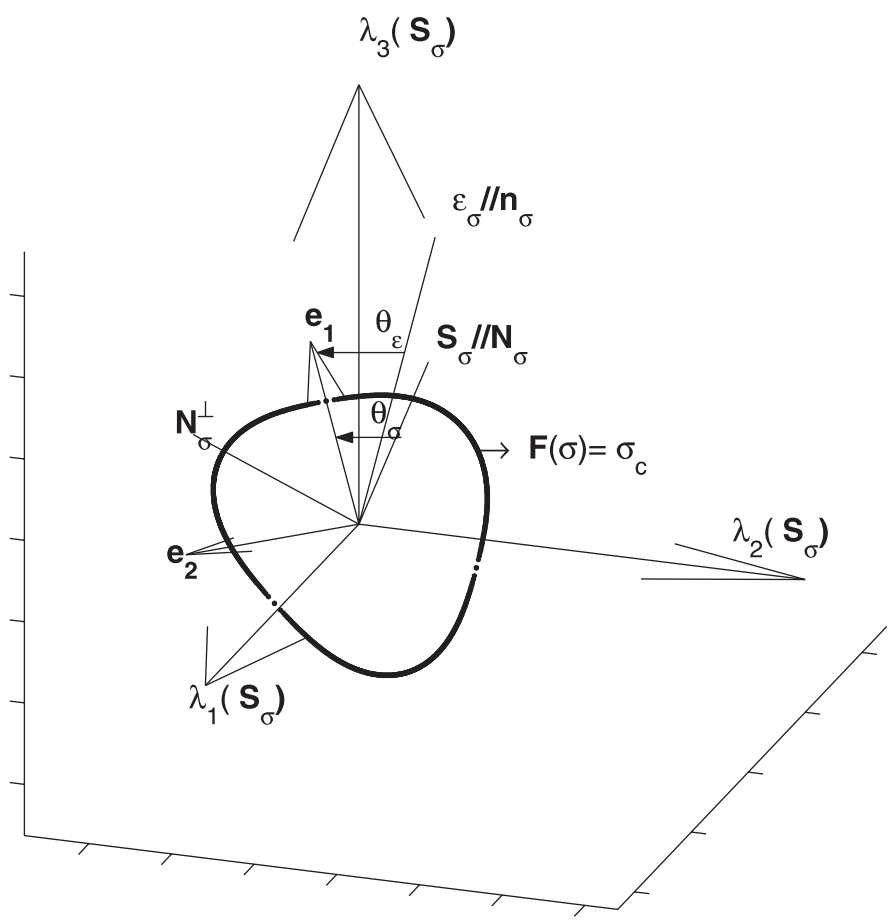

Figure 1. Deviatoric stress section: definition of angle $\theta_{\sigma}, \theta_{\epsilon}$ and yield surface $F(\boldsymbol{\sigma})=\sigma_{c}$.

In the first part, yield criteria for isotropic materials will be stated with a special attention devoted to the convexity of the yield surface of phase transformation initiation in the stress space $\boldsymbol{\sigma}$. The second part will concern the transport of these surfaces in the space of effective transformation strain $\epsilon_{m}$ associated to the produced martensitic phase.

Some applications on NiTi and $\mathrm{Cu}-\mathrm{Al}-\mathrm{Be}$ isotropic SMA end the paper.

\section{CONSIDERATIONS ON THE YIELD CRITERIA}

\subsection{General Formulations and Haigh-Westergaard Representation}

As the analysis is restricted to isotropic behavior, the Haigh-Westergaard representation is used (see Figure 1).

Let us consider the second order symmetric tensor $\boldsymbol{\sigma}$ (defined in $\mathbb{R}^{3}$ ) and the deviatoric tensor $\mathbf{S}_{\sigma}$ defined by 


$$
\mathbf{S}_{\sigma} \equiv \boldsymbol{\sigma}-\frac{1}{3} \operatorname{tr}(\boldsymbol{\sigma}) \mathbf{1}
$$

where $\mathbf{1}$ is the identity tensor. Two dots denote the scalar product and $\operatorname{tr}($.$) denotes the trace$ operator, so that $\mathbf{a}: \mathbf{b} \equiv \operatorname{tr}\left(\mathbf{a}^{T} \mathbf{b}\right)$ for every second order tensor $\mathbf{a}$ and $\mathbf{b}$ and $|\mathbf{a}| \equiv \sqrt{\operatorname{tr}\left(\mathbf{a}^{T} \mathbf{a}\right)}$.

In a classical way, the Huber-Von Mises equivalent stress $\overline{\boldsymbol{\sigma}}$ is defined by

$$
\overline{\boldsymbol{\sigma}} \equiv \kappa\left|\mathbf{S}_{\sigma}\right|,
$$

with the normalization parameter $\kappa \equiv \sqrt{\frac{3}{2}}$.

The yield surface equation of phase transformation initiation reads

$$
\mathcal{F}(\boldsymbol{\sigma}) \equiv \overline{\boldsymbol{\sigma}} f\left(x_{\sigma}\right)=\sigma_{c},
$$

where $f>0$, is a smooth function (the second derivative is continuous) defined on the interval $I_{x}$ (which will be defined later). In fact, $f$ is some "correction function" which allows to take into account the asymmetry between tension and compression called SD effect (stress differential effect), see Raniecki and Mroz [3]. In Podgorski [4, 5], $f$ is referred to as the "shape function".

The positive quantity $\sigma_{c}$ is the yield phase transformation initiation value and is given by

$$
\sigma_{c} \equiv b\left(T-M_{S}^{0}\right)
$$

where $M_{S}^{0}$ is the martensite start temperature at stress-free state.

The interval $I_{x}$ is defined following the choice of the argument $x_{\sigma}$ of $f$. In fact, there are at least three possibilities for $x_{\sigma}$ :

1. The Lode invariant $y_{\sigma}$, chosen for instance by Raniecki and Mroz [3], which is an homogeneous function of degree zero of stress deviator

$$
x_{\sigma} \equiv y_{\sigma} \equiv 4 \operatorname{det}\left(\mathbf{N}_{\sigma}\right) \in I_{y_{\sigma}} \equiv[-1,1]
$$

and where $\mathbf{N}_{\sigma}$ is the reduced deviatoric part of $\boldsymbol{\sigma}$ given by

$$
\mathbf{N}_{\sigma} \equiv \frac{\kappa^{2}}{\overline{\mathbf{\sigma}}} \mathbf{S}_{\sigma}
$$

with

$$
\mathbf{N}_{\sigma}: \mathbf{N}_{\sigma}=\kappa^{2} .
$$

2. The Lode angle $\theta_{\sigma}$ (see [6]) defined by

$$
x_{\sigma} \equiv \theta_{\sigma} \equiv \frac{1}{3} \arccos y_{\sigma} \in I_{\theta_{\sigma}} \equiv\left[0, \frac{\pi}{3}\right] .
$$


This latter variable was chosen as variable by Bigoni and Piccolroaz [7] together with

$$
f\left(x_{\sigma}\right) \equiv \frac{1}{f_{*}\left(\theta_{\sigma}\right)} .
$$

as the shape function.

3. In the present paper, a third expression of $x_{\sigma}$ is introduced in order to simplify the calculations and the formulations, notably the one associated with the convexity condition. We take the greatest eigenvalue of $\mathbf{N}_{\sigma}$, i.e.

$$
x_{\sigma} \equiv \rho_{\sigma} \equiv \cos \left(\frac{1}{3} \arccos y_{\sigma}\right) \in I_{\rho_{\sigma}} \equiv\left[\frac{1}{2}, 1\right] \text {. }
$$

\subsection{Yield Condition Resolution in Terms of Admissible Stress}

Let us recall the yield equation (1):

$$
\overline{\boldsymbol{\sigma}}=\frac{\sigma_{c}}{f\left(x_{\sigma}\right)}
$$

the solution of which can be written as

$$
\boldsymbol{\sigma}=\frac{1}{3} \operatorname{tr}(\boldsymbol{\sigma}) \mathbf{1}+\frac{\sigma_{c}}{f\left(x_{\sigma}\right)} \kappa^{-2} \mathbf{N}_{\sigma}
$$

One can show that $\mathbf{N}_{\sigma}$ can be split as (see (55) in Proposition 1)

$$
\mathbf{N}_{\sigma}=\rho_{\sigma} \mathbf{e}_{1}^{\mathbf{R}_{\sigma}}-\left(1-\rho_{\sigma}^{2}\right)^{\frac{1}{2}} \mathbf{e}_{2}^{\mathbf{R}_{\sigma}}
$$

where $\rho_{\sigma} \equiv \rho\left(x_{\sigma}\right)$, with $\rho$ a bijective function which maps $I_{x}$ on

$$
I \equiv\left[\frac{1}{2}, 1\right]
$$

and which is defined by

$$
\rho(x) \equiv \begin{cases}\cos \left(\frac{1}{3} \arccos (x)\right) & \text { for the first choice }\left(x=y_{\sigma}\right) \\ \cos (x) & \text { for the second choice }\left(x=\theta_{\sigma}\right) \\ x & \text { for the third choice }\left(x=\rho_{\sigma}\right)\end{cases}
$$

Note that the reciprocal application gives 


$$
x(\rho)= \begin{cases}4 \rho^{3}-3 \rho & \text { for the first choice } \\ \arccos (\rho) & \text { for the second choice } \\ \rho & \text { for the third choice. }\end{cases}
$$

In (9), $\mathbf{e}_{1}^{\mathbf{R}_{\sigma}}$ and $\mathbf{e}_{2}^{\mathbf{R}_{\sigma}}$ are two orthogonal elementary deviators associated to $\boldsymbol{\sigma}$ by

$$
\mathbf{e}_{1}^{\mathbf{R}_{\sigma}} \equiv \mathbf{R}_{\sigma} \mathbf{e}_{1} \mathbf{R}_{\sigma}^{T} \quad \text { and } \quad \mathbf{e}_{2}^{\mathbf{R}_{\sigma}} \equiv \mathbf{R}_{\sigma} \mathbf{e}_{2} \mathbf{R}_{\sigma}^{T}
$$

where $\mathbf{R}_{\sigma}$ is the rotation matrix transforming $\boldsymbol{\sigma}$ in its diagonal form

$$
\begin{gathered}
\Lambda_{\sigma} \equiv \mathbf{R}_{\sigma}^{T} \boldsymbol{\sigma} \mathbf{R}_{\sigma}=\left(\begin{array}{ccc}
\lambda_{1}(\boldsymbol{\sigma}) & 0 & 0 \\
0 & \lambda_{2}(\boldsymbol{\sigma}) & 0 \\
0 & 0 & \lambda_{3}(\boldsymbol{\sigma})
\end{array}\right) \\
\lambda_{1}(\boldsymbol{\sigma}) \leq \lambda_{2}(\boldsymbol{\sigma}) \leq \lambda_{3}(\boldsymbol{\sigma})
\end{gathered}
$$

and

$$
\mathbf{e}_{1} \equiv\left(\begin{array}{ccc}
-\frac{1}{2} & 0 & 0 \\
0 & -\frac{1}{2} & 0 \\
0 & 0 & 1
\end{array}\right), \quad \mathbf{e}_{2} \equiv\left(\begin{array}{ccc}
\frac{\sqrt{3}}{2} & 0 & 0 \\
0 & -\frac{\sqrt{3}}{2} & 0 \\
0 & 0 & 0
\end{array}\right)
$$

One can easily verify that

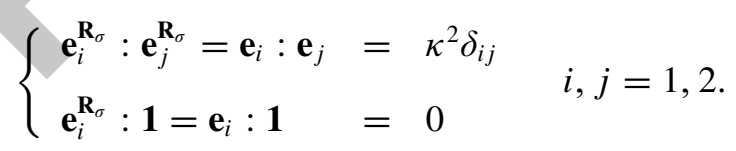

Note that the Lode $\theta_{\sigma}$ is the angle between $\mathbf{N}_{\sigma}$ and $\mathbf{e}_{1}^{\mathbf{R}_{\sigma}}$ see (56) and Figure 1. Moreover $\Lambda_{\sigma}$, the solution of equation (1), can be written as

$$
\Lambda_{\sigma}=\frac{1}{3} \operatorname{tr}\left(\Lambda_{\sigma}\right) \mathbf{1}+\frac{\sigma_{c}}{f(x)} \kappa^{-2}\left(\rho \mathbf{e}_{1}-\left(1-\rho^{2}\right)^{\frac{1}{2}} \mathbf{e}_{2}\right) \forall \rho \in I,
$$

$x$ being linked to $\rho$ by the relations (11).

Therefore the eigenvalues $\lambda_{k}$ of the reduced stress tensor $\frac{\boldsymbol{\sigma}}{\sigma_{c}}$ are solutions of the following system: 


$$
\left\{\begin{array}{l}
2 \lambda_{1}-\lambda_{2}-\lambda_{3}=\rho_{1} \equiv \frac{1}{f(x)}\left(-\rho-\sqrt{3}\left(1-\rho^{2}\right)^{\frac{1}{2}}\right) \\
2 \lambda_{2}-\lambda_{3}-\lambda_{1}=\rho_{2} \equiv \frac{1}{f(x)}\left(-\rho+\sqrt{3}\left(1-\rho^{2}\right)^{\frac{1}{2}}\right) \\
2 \lambda_{3}-\lambda_{1}-\lambda_{2}=\rho_{3} \equiv \frac{2}{f(x)} \rho,
\end{array}\right.
$$

for all $\rho \in I$.

Now let us examine the dual space associated to the martensite strain.

\section{TENSOR OF EFFECTIVE TRANSFORMATION STRAIN OF MARTENSITE}

The concept of "effective transformation strain of martensite" has been introduced by Sadjadpour and Bhattacharya [8]. This strain tensor called $\varepsilon_{m}$ is the average transformation strain of the different variants of martensite averaged over a representative volume containing multiple grains, after the material has formed an allowable microstructure.

For proportional loadings the experiments show that the phase transformation strain rate is normal to the yield surface $\mathcal{F}(\boldsymbol{\sigma})=\sigma_{c}$ (see Bouvet et al. [9]).

Thus one can assume that $\varepsilon_{m}(\boldsymbol{\sigma})$ admits a potential $\mathcal{F}(\boldsymbol{\sigma})>0$, which means

$$
\boldsymbol{\epsilon}_{m} \equiv \gamma \mathcal{F}^{\prime}(\boldsymbol{\sigma})
$$

Let us introduce the reduced strain tensor

$$
\boldsymbol{\epsilon}_{\sigma} \equiv \frac{\boldsymbol{\epsilon}_{m}}{\gamma}=\mathcal{F}^{\prime}(\boldsymbol{\sigma})
$$

We give two decompositions for the tensor $\epsilon_{\sigma}$ as follows.

\subsection{First Decomposition}

The first one is given for $x=y_{\sigma}, \theta_{\sigma}$ or $\rho_{\sigma}$ (see (59) in Proposition 2):

$$
\boldsymbol{\epsilon}_{\sigma}=f(x) \mathbf{N}_{\sigma}+a(x) f^{\prime}(x) \mathbf{N}_{\sigma}^{\perp}
$$

where

$$
\mathbf{N}_{\sigma}^{\perp} \equiv\left(1-\rho_{\sigma}^{2}\right)^{\frac{1}{2}} \mathbf{e}_{1}^{\mathbf{R}_{\sigma}}+\rho_{\sigma} \mathbf{e}_{2}^{\mathbf{R}_{\sigma}}
$$

is an orthogonal matrix to $\mathbf{N}_{\sigma}$ and 


$$
a(x) \equiv \frac{\mathrm{d} x}{\mathrm{~d} \rho}\left(1-|\rho(x)|^{2}\right)^{\frac{1}{2}}=\left\{\begin{array}{lll}
3\left(1-x^{2}\right)^{\frac{1}{2}} & \text { if } \quad x=y_{\sigma} \\
-1 & \text { if } \quad x=\theta_{\sigma} \\
\left(1-x^{2}\right)^{\frac{1}{2}} & \text { if } \quad x=\rho_{\sigma}
\end{array}\right.
$$

that is

$$
\boldsymbol{\epsilon}_{\sigma}= \begin{cases}f\left(y_{\sigma}\right) \mathbf{N}_{\sigma}+3\left(1-y_{\sigma}^{2}\right)^{\frac{1}{2}} f^{\prime}\left(y_{\sigma}\right) \mathbf{N}_{\sigma}^{\perp} & \text { for } x=y_{\sigma} \\ f\left(\theta_{\sigma}\right) \mathbf{N}_{\sigma}-f^{\prime}\left(\theta_{\sigma}\right) \mathbf{N}_{\sigma}^{\perp} & \text { for } x=\theta_{\sigma} \\ f\left(\rho_{\sigma}\right) \mathbf{N}_{\sigma}+\left(1-\rho_{\sigma}\right)^{\frac{1}{2}} f^{\prime}\left(\rho_{\sigma}\right) \mathbf{N}_{\sigma}^{\perp} & \text { for } x=\rho_{\sigma} .\end{cases}
$$

Note that the orthogonal matrix $\mathbf{N}_{\sigma}^{\perp}$ can be expressed as a function of $\mathbf{N}_{\sigma}$. In fact, $\mathbf{N}_{\sigma}^{\perp}$ is defined by

$$
\mathbf{N}_{\sigma}^{\perp}=\mathbf{R}_{\sigma} \widetilde{\Lambda}_{\sigma}^{\perp} \mathbf{R}_{\sigma}^{\mathrm{T}}
$$

where

$$
\widetilde{\Lambda}_{\sigma}^{\perp}=\left(1-\rho_{\sigma}^{2}\right)^{\frac{1}{2}} \mathbf{e}_{1}+\rho_{\sigma} \mathbf{e}_{2}
$$

So for $\rho_{\sigma}=\cos \left(\frac{1}{3} \arccos \left(y_{\sigma}\right)\right)$, we have

$$
\widetilde{\Lambda}_{\sigma}^{\perp}=\operatorname{diag}\left(\sin \left(\frac{1}{3} \arccos \left(y_{\sigma}\right)+\frac{2 k}{3} \pi\right)\right) .
$$

By using the trigonometrical identity

$$
\sin (y) \sin \left(\frac{1}{3} y\right)=2 \cos ^{2}\left(\frac{1}{3} y\right)-1-\cos (y) \cos \frac{1}{3} y,
$$

for $y=\arccos \left(y_{\sigma}\right)+2 k \pi$, one finally obtains the relation between the eigenvalues of $\mathbf{N}_{\sigma}$ and $\mathbf{N}_{\sigma}^{\perp}$

$$
\left(1-y_{\sigma}^{2}\right)^{\frac{1}{2}} \lambda_{k}\left(\mathbf{N}_{\sigma}^{\perp}\right)=2\left|\lambda_{k}\left(\mathbf{N}_{\sigma}\right)\right|^{2}-1-y_{\sigma} \lambda_{k}\left(\mathbf{N}_{\sigma}\right) .
$$

And one recovers the classical expression (see [3])

$$
\left(1-y_{\sigma}^{2}\right)^{\frac{1}{2}} \mathbf{N}_{\sigma}^{\perp}=2 \mathbf{N}_{\sigma}^{2}-\mathbf{1}-y_{\sigma} \mathbf{N}_{\sigma}
$$

Moreover the substitution of $y_{\sigma}$ by $\cos \left(3 \theta_{\sigma}\right)$ leads to the equivalent expression in $\theta_{\sigma}$ terms obtained in [7]:

$$
\sin \left(3 \theta_{\sigma}\right) \mathbf{N}_{\sigma}^{\perp}=2 \mathbf{N}_{\sigma}^{2}-\mathbf{1}-\cos \left(3 \theta_{\sigma}\right) \mathbf{N}_{\sigma} .
$$




\subsection{Second Decomposition}

This decomposition is done in the basis $\left\{\mathbf{e}_{1}^{\mathbf{R}_{\sigma}}, \mathbf{e}_{2}^{\mathbf{R}_{\sigma}}\right\}$. One obtains easily (see Proposition 3)

$$
\boldsymbol{\epsilon}_{\sigma}=f_{1}\left(\rho_{\sigma}\right) \mathbf{e}_{1}^{\mathbf{R}_{\sigma}}-f_{2}\left(\rho_{\sigma}\right) \mathbf{e}_{2}^{\mathbf{R}_{\sigma}}
$$

where

$$
\left\{\begin{array}{l}
f_{1}(\rho)=\left(1-\rho^{2}\right) \frac{\mathrm{d} f}{\mathrm{~d} \rho}+\rho f(x) \\
f_{2}(\rho)=\left(1-\rho^{2}\right)^{\frac{1}{2}} g(\rho)
\end{array}\right.
$$

with $x=x(\rho)($ see (11)) and

$$
g(\rho) \equiv-\rho \frac{\mathrm{d} f}{\mathrm{~d} \rho}+f(x)
$$

Now let us examine the convexity conditions of the yield function $\mathcal{F}$.

\section{CONVEXITY CONDITIONS}

In order to establish the convexity conditions, $g$ and $\frac{\mathrm{d}^{2} f}{\mathrm{~d} \rho^{2}}$ must be continuous functions on $I$ (see (67) and (68) in Proposition 4). So, for $x=y_{\sigma}$ or $x=\rho_{\sigma}$ where $g$ and $\frac{\mathrm{d}^{2} f}{\mathrm{~d} \rho^{2}}$ are continuous on $I$ without any additional hypothesis, it is shown that the $\mathcal{F}$ function is convex if and only if (see Proposition 5)

$$
\left\{\begin{array}{l}
g(\rho) \geq 0 \\
\frac{\mathrm{d}^{2} f}{\mathrm{~d} \rho^{2}}(\rho) \geq 0
\end{array}\right.
$$

for all $\rho \in I$.

One can note that the condition (22) implies

$$
\frac{\mathrm{d} f_{1}}{\mathrm{~d} \rho}=g(\rho)+\left(1-\rho^{2}\right) \frac{\mathrm{d}^{2} f}{\mathrm{~d} \rho^{2}} \geq 0 \forall \rho \in I,
$$

and

$$
\left\{\begin{array}{l}
f_{2}=\left(1-\rho^{2}\right)^{\frac{1}{2}} g(\rho) \quad \geq 0 \quad \forall \rho \in I \\
\frac{\mathrm{d} f_{2}}{\mathrm{~d} \rho}=-\rho\left(1-\rho^{2}\right)^{-\frac{1}{2}} \frac{\mathrm{d} f_{1}}{\mathrm{~d} \rho} \leq 0 \quad \forall \rho \in\left[\frac{1}{2}, 1[.\right.
\end{array}\right.
$$


Let us point out that (22) is equivalent to

$$
\left\{\begin{array}{l}
g(1) \geq 0 \\
\frac{\mathrm{d}^{2} f}{\mathrm{~d} \rho^{2}}(\rho) \geq 0
\end{array}\right.
$$

due to the fact that $g$ is decreasing. Indeed we have

$$
\frac{\mathrm{d}^{2} f}{\mathrm{~d} \rho^{2}}=-\rho^{-1} \frac{\mathrm{d} g}{\mathrm{~d} \rho} \geq 0 .
$$

Thus the condition (22) is also equivalent to

$$
\left\{\begin{array}{l}
g(\rho) \geq 0 \\
\frac{\mathrm{d} g}{\mathrm{~d} \rho}(\rho) \leq 0
\end{array}\right.
$$

for all $\rho \in I$. This latter condition is very useful for the numerical verifications.

Now we are going to explicit the convexity conditions following the choice of the variables.

\subsection{Conditions in Terms of $\rho_{\sigma}$}

The inequalities in (25) for $x=\rho_{\sigma}$ are the simplest because $\rho=x$. One obtains

$$
\begin{cases}f(1)-f^{\prime}(1) & \geq 0 \\ f^{\prime \prime}(\rho) & \geq 0\end{cases}
$$

for all $\rho \in\left[\frac{1}{2}, 1\right]$.

\subsection{Conditions in Terms of $y_{\sigma}$}

When $f$ is a function of $x=y_{\sigma}$ with $\rho=\cos \left(\frac{1}{3} \arccos \left(y_{\sigma}\right)\right)$, the expression of the sufficient and necessary conditions for the convexity of $\mathcal{F}$ is more complicated. In fact it verifies (see (67) and (68))

$$
\left\{\begin{array}{l}
g(\rho)=f(y)-3 \rho\left(4 \rho^{2}-1\right) f^{\prime}(y) \geq 0 \\
\frac{\mathrm{d}^{2} f}{\mathrm{~d} \rho^{2}}=24 \rho f^{\prime}(y)+9\left(4 \rho^{2}-1\right)^{2} f^{\prime \prime}(y) \geq 0
\end{array}\right.
$$


for all $y \in[-1,1]$, or by using (25)

$$
\begin{cases}g(1)=f(1)-9 f^{\prime}(1) & \geq 0 \\ \frac{\mathrm{d}^{2} f}{\mathrm{~d} \rho^{2}}=24 \rho f^{\prime}(y)+9\left(4 \rho^{2}-1\right)^{2} f^{\prime \prime}(y) \geq 0 .\end{cases}
$$

Note that due to the identities $4 \rho^{3}-3 \rho=y$ and $\left(1-\rho^{2}\right)\left(4 \rho^{2}-1\right)^{2}=1-y^{2},(23)$ becomes simply

$$
\frac{\mathrm{d} f_{1}}{\mathrm{~d} \rho}=\frac{1}{9} f-y f^{\prime}(y)+\left(1-y^{2}\right) f^{\prime \prime}(y) \geq 0 \quad \forall y \in[-1,1],
$$

which is the convexity condition given by Raniecki and Mroz [3]. It is clear that this latter condition is just necessary but not sufficient as will be seen below.

\subsection{Conditions in Terms of $\theta_{\sigma}$}

A supplementary condition on $f$ is required, namely the continuity of $g$ and of $\frac{\mathrm{d}^{2} f}{\mathrm{~d} \rho^{2}}$ at the neighborhood of $\theta=0$ (see (67) and (68)).

It results from (22) for $x=\theta=\arccos (\rho)$ (see (67) and (68)) that

$$
\left\{\begin{array}{l}
g(\rho)=f(\theta)+\frac{\cos (\theta)}{\sin (\theta)} f^{\prime}(\theta) \geq 0 \\
\frac{\mathrm{d}^{2} f}{\mathrm{~d} \rho^{2}}=-\frac{\cos (\theta)}{\sin ^{3} \theta} f^{\prime}(\theta)+\frac{1}{\sin ^{2}(\theta)} f^{\prime \prime}(\theta) \geq 0
\end{array}\right.
$$

for all $\left.\theta \in] 0, \frac{\pi}{3}\right]$.

Due to the continuity at $\theta=0$, the above system is equivalent to

$$
\left\{\begin{array}{l}
\sin (\theta) f+\cos (\theta) \frac{\mathrm{d} f}{\mathrm{~d} \theta} \geq 0 \\
-\cos (\theta) \frac{\mathrm{d} f}{\mathrm{~d} \theta}+\sin \theta \frac{\mathrm{d}^{2} f}{\mathrm{~d} \theta^{2}} \geq 0
\end{array}\right.
$$

for all $\theta \in\left[0, \frac{\pi}{3}\right]$.

These necessary conditions are not practical. However, as in the previous situation, the condition (23) is very simple:

$$
\frac{\mathrm{d} f_{1}}{\mathrm{~d} \rho}=f(\theta)+f^{\prime \prime}(\theta) \geq 0 \quad \forall \theta \in\left[0, \frac{\pi}{3}\right] .
$$

If now, instead of $f$ one uses its inverse $f_{*} \equiv f^{-1}$, then in this case, the condition (32) becomes 


$$
f_{*}^{2}+2 f_{*}^{\prime 2}-f_{*}^{\prime \prime} f_{*} \geq 0
$$

We thus recover the condition established by Bigoni and Piccolroaz, see [7].

\subsection{Counter-example}

The necessary conditions established by Raniecki and Mroz [3], Bigoni and Piccolroaz [7] and (30), (32) and (33) which come from (23) are not sufficient. In order to convince the reader let us give a counter-example:

$$
f=2-\cos ^{2}(\theta)=2-\cos ^{2}\left(\frac{1}{3} \arccos y\right)=2-\rho^{2}>0 \quad \forall \rho \in\left[\frac{1}{2}, 1\right] .
$$

The condition (23)

$$
\frac{\mathrm{d} f_{1}}{\mathrm{~d} \rho}=f-\rho \frac{\mathrm{d} f}{\mathrm{~d} \rho}+\left(1-\rho^{2}\right) \frac{\mathrm{d}^{2} f}{\mathrm{~d} \rho^{2}}=3 \rho^{2}>0,
$$

is fulfilled but not the positivity of the second derivative of $f$ :

$$
\frac{\mathrm{d}^{2} f}{\mathrm{~d} \rho^{2}}=-2<0
$$

Let us give a concrete example where the convexity condition is not fulfilled. Take $\mathbf{a}, \mathbf{b}, \mathbf{c}$ such that

$$
\begin{aligned}
& \left.\mathbf{a}=\kappa\left(\begin{array}{ccc}
6 & 0 & 0 \\
0 & -3 & 0 \\
0 & 0 & 0
\end{array}\right) \Rightarrow \begin{array}{l}
\overline{\mathbf{a}}=\sqrt{42} \kappa^{2} \\
\rho_{\mathbf{a}}=\frac{5}{\sqrt{42}} \kappa
\end{array}\right\} \Rightarrow f\left(\rho_{\mathbf{a}}\right)=\frac{31}{28}, \\
& \left.\mathbf{b}=\kappa\left(\begin{array}{ccc}
-6 & 0 & 0 \\
0 & -9 & 0 \\
0 & 0 & 0
\end{array}\right) \Rightarrow \begin{array}{l}
\overline{\mathbf{b}}=\sqrt{42} \kappa^{2} \\
\rho_{\mathbf{b}}=\frac{5}{\sqrt{42}} \kappa
\end{array}\right\} \Rightarrow f\left(\rho_{\mathbf{b}}\right)=\frac{31}{28}
\end{aligned}
$$

and $\mathbf{c}=\frac{1}{2}(\mathbf{a}+\mathbf{b})$

$$
\left.\mathbf{c}=\kappa\left(\begin{array}{ccc}
0 & 0 & 0 \\
0 & -6 & 0 \\
0 & 0 & 0
\end{array}\right) \Rightarrow \begin{array}{l}
\overline{\mathbf{c}}=2 \sqrt{6} \kappa^{2} \\
\rho_{\mathbf{c}}=\frac{1}{\sqrt{6}} \kappa
\end{array}\right\} \Rightarrow f\left(\rho_{\mathbf{c}}\right)=\frac{7}{4} .
$$




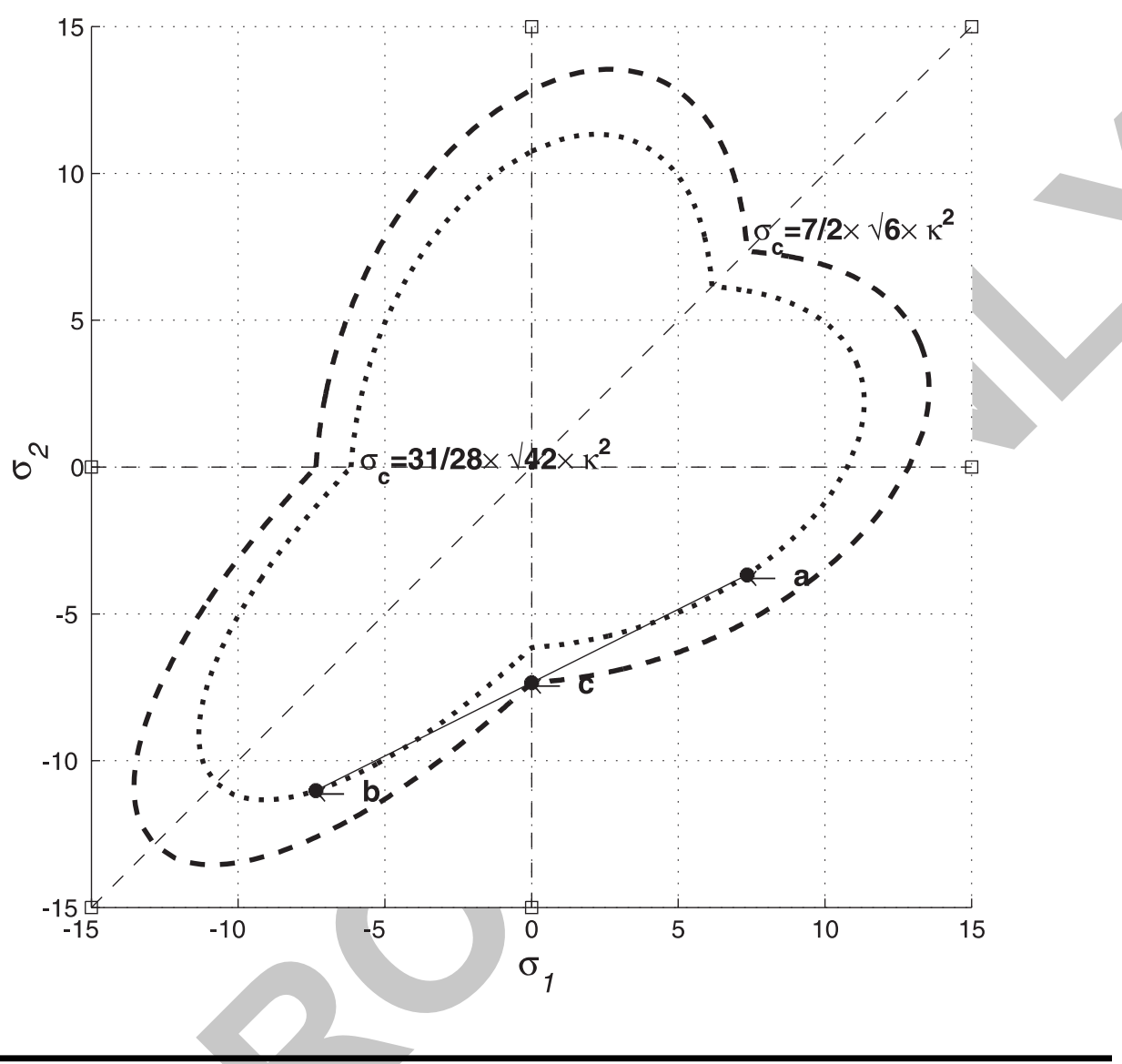

Figure 2. Non convex yield surface(counter example).

One finds

$$
\begin{aligned}
& \mathcal{F}(\mathbf{a})=\mathcal{F}(\mathbf{b})=\frac{31}{28} \sqrt{42} \kappa^{2}-\sigma_{c} \text { and } \\
& \mathcal{F}(\mathbf{c})=\frac{7}{2} \sqrt{6} \kappa^{2}-\sigma_{c}
\end{aligned}
$$

and it is clear that

$$
\mathcal{F}(\mathbf{c}) \not \leq \frac{1}{2}(\mathcal{F}(\mathbf{a})+\mathcal{F}(\mathbf{b})) \text {. }
$$

This case is illustrated in Figure 2. In the next section, we return to the expression of the phase transformation strain tensor $\varepsilon_{\sigma}$. 


\section{REDUCED SHAPE OF THE PHASE TRANSFORMATION STRAIN TENSOR $\varepsilon_{\sigma}$}

As before one determines the reduced shape of $\epsilon_{\sigma}$, with the reduced formulae (4):

$$
\mathbf{n}_{\sigma} \equiv \frac{\kappa^{2}}{\bar{\epsilon}_{\sigma}} \boldsymbol{\epsilon}_{\sigma}=\frac{1}{\delta_{f}\left(x_{\sigma}\right)} \boldsymbol{\epsilon}_{\sigma}
$$

where

$$
\overline{\boldsymbol{\epsilon}}_{\sigma} \equiv \kappa\left|\boldsymbol{\epsilon}_{\sigma}\right|=\kappa^{2} \delta_{f}\left(x_{\sigma}\right)
$$

with

$$
\delta_{f}\left(x_{\sigma}\right) \equiv\left(\left|f\left(x_{\sigma}\right)\right|^{2}+\left|a\left(x_{\sigma}\right)\right|^{2}\left|f^{\prime}\left(x_{\sigma}\right)\right|^{2}\right)^{\frac{1}{2}}=\left(\left|f_{1}\left(\rho_{\sigma}\right)\right|^{2}+\left|f_{2}\left(\rho_{\sigma}\right)\right|^{2}\right)^{\frac{1}{2}},
$$

$x_{\sigma}$ and $\rho_{\sigma}$ being linked in an univocal sense by (11).

$\mathbf{n}_{\sigma}$ can be written as

$$
\mathbf{n}_{\sigma}=\frac{f\left(x_{\sigma}\right)}{\delta_{f}\left(x_{\sigma}\right)} \mathbf{N}_{\sigma}+\frac{a\left(x_{\sigma}\right) f^{\prime}\left(x_{\sigma}\right)}{\delta_{f}\left(x_{\sigma}\right)} \mathbf{N}_{\sigma}^{\perp}=\frac{f_{1}\left(\rho_{\sigma}\right)}{\delta_{f}\left(x_{\sigma}\right)} \mathbf{e}_{1}^{\mathbf{R}_{\sigma}}-\frac{f_{2}\left(\rho_{\sigma}\right)}{\delta_{f}\left(x_{\sigma}\right)} \mathbf{e}_{2}^{\mathbf{R}_{\sigma}} .
$$

From now on, we will always suppose that the convexity conditions (22) are satisfied.

\subsection{First Decomposition}

The function $f_{2}$, as defined in (20), being positive, one can write

$$
\mathbf{n}_{\sigma}=\rho_{\varepsilon} \mathbf{e}_{1}^{\mathbf{R}_{\sigma}}-\left(1-\rho_{\varepsilon}^{2}\right)^{\frac{1}{2}} \mathbf{e}_{2}^{\mathbf{R}_{\sigma}},
$$

where

$$
\rho_{\varepsilon}=\frac{f_{1}\left(\rho_{\sigma}\right)}{\delta_{f}\left(x_{\sigma}\right)} \quad \text { and } \quad \frac{f_{2}\left(\rho_{\sigma}\right)}{\delta_{f}\left(x_{\sigma}\right)}=\left(1-\rho_{\varepsilon}^{2}\right)^{\frac{1}{2}} .
$$

In the above, $\rho_{\varepsilon}$ denotes the greatest eigenvalue of $\mathbf{n}_{\sigma}$ characterized by (56)

$$
\rho_{\varepsilon}=\kappa^{-2}\left(\mathbf{n}_{\sigma}: \mathbf{e}_{1}^{\mathbf{R}_{\sigma}}\right) .
$$

Just as for $\mathbf{N}_{\sigma}$ (see (10)), we have 


$$
\rho_{\epsilon}= \begin{cases}\cos \left(\frac{1}{3} \arccos \left(x_{\epsilon}\right)\right) & \text { for } x_{\epsilon}=y_{\epsilon} \equiv 4 \operatorname{det}\left(\mathbf{n}_{\sigma}\right) \\ \cos \left(x_{\epsilon}\right) & \text { for } x_{\epsilon}=\theta_{\epsilon} \equiv \frac{1}{3} \arccos y_{\epsilon} \\ x_{\epsilon} & \text { for } x_{\epsilon}=\rho_{\epsilon} .\end{cases}
$$

In order to make the link with $\boldsymbol{\sigma}$, we admit that

$$
\rho_{\epsilon}\left(x_{\epsilon}\right) \equiv \begin{cases}\cos \left(\frac{1}{3} \arccos \left(y_{\epsilon}\right)\right) & \text { if } x_{\epsilon}=y_{\epsilon} \\ \cos \left(\theta_{\epsilon}\right) & \text { if } x_{\epsilon}=\theta_{\epsilon} \\ \rho_{\epsilon} & \text { if } x_{\epsilon}=\rho_{\epsilon} .\end{cases}
$$

The reciprocal application is such that

$$
x_{\epsilon}= \begin{cases}4 \rho_{\epsilon}^{3}-3 \rho_{\epsilon} & \text { for } x_{\epsilon}=y_{\epsilon} \\ \arccos \left(\rho_{\epsilon}\right) & \text { for } x_{\epsilon}=\theta_{\epsilon} \\ \rho_{\epsilon} & \text { for } x_{\epsilon}=\rho_{\epsilon} .\end{cases}
$$

\subsection{Second Decomposition}

The reduced tensor $\mathbf{n}_{\sigma}$ in the basis $\mathbf{N}_{\sigma}$ and $\mathbf{N}_{\sigma}^{\perp}$ can be written as

$$
\mathbf{n}_{\sigma}=\cos \chi_{\sigma} \mathbf{N}_{\sigma}+\sin \chi_{\sigma} \mathbf{N}_{\sigma}^{\perp}
$$

where $\left.\chi_{\sigma} \in\right]-\frac{\pi}{2}, \frac{\pi}{2}[$ is such that

$$
\cos \chi_{\sigma}=\frac{f\left(x_{\sigma}\right)}{\delta_{f}\left(x_{\sigma}\right)}>0 \text { and } \quad \sin \chi_{\sigma}=\frac{a\left(x_{\sigma}\right) f^{\prime}\left(x_{\sigma}\right)}{\delta_{f}\left(x_{\sigma}\right)}
$$

i.e.

$$
\chi_{\sigma}=\arctan \left(\frac{a\left(x_{\sigma}\right) f^{\prime}\left(x_{\sigma}\right)}{f\left(x_{\sigma}\right)}\right)=\arcsin \left(\frac{a\left(x_{\sigma}\right) f^{\prime}\left(x_{\sigma}\right)}{\delta_{f}\left(x_{\sigma}\right)}\right) .
$$

The angle $\chi_{\sigma}$ is linked to $\rho_{\epsilon}$ (see (66) and (65)) by

$$
\left\{\begin{array}{l}
\sin \chi_{\sigma}=\left(1-\rho_{\sigma}^{2}\right)^{\frac{1}{2}} \rho_{\varepsilon}-\rho_{\sigma}\left(1-\rho_{\varepsilon}^{2}\right)^{\frac{1}{2}} \\
\cos \chi_{\sigma}=\rho_{\sigma} \rho_{\varepsilon}+\left(1-\rho_{\sigma}^{2}\right)^{\frac{1}{2}}\left(1-\rho_{\varepsilon}^{2}\right)^{\frac{1}{2}}
\end{array}\right.
$$


and

$$
\left\{\begin{aligned}
\rho_{\varepsilon} & =\rho_{\sigma} \cos \chi_{\sigma}+\left(1-\rho_{\sigma}^{2}\right)^{\frac{1}{2}} \sin \chi_{\sigma} \\
\left(1-\rho_{\varepsilon}^{2}\right)^{\frac{1}{2}} & =\left(1-\rho_{\sigma}^{2}\right)^{\frac{1}{2}} \cos \chi_{\sigma}-\rho_{\sigma} \sin \chi_{\sigma} .
\end{aligned}\right.
$$

As $\rho_{\sigma}=\cos \left(\theta_{\sigma}\right)$, the system becomes

$$
\left\{\begin{array}{rrr}
\rho_{\varepsilon} & = & \cos \left(\theta_{\sigma}-\chi_{\sigma}\right) \\
\left(1-\rho_{\varepsilon}^{2}\right)^{\frac{1}{2}} & = & \sin \left(\theta_{\sigma}-\chi_{\sigma}\right) \geq 0,
\end{array}\right.
$$

or more simply written as

$$
\left\{\begin{array}{l}
\theta_{\varepsilon}=\theta_{\sigma}-\chi_{\sigma} \\
\rho_{\varepsilon}=\cos \left(\arccos \rho_{\sigma}-\chi_{\sigma}\right) \\
y_{\epsilon}=\cos \left(\arccos y_{\sigma}-3 \chi_{\sigma}\right) .
\end{array}\right.
$$

\subsection{Univocal Correspondence Between $\sigma$ and $\epsilon_{\sigma}$}

The correspondence between $x_{\epsilon}=y_{\epsilon}, \theta_{\epsilon}$ or $\rho_{\varepsilon}$ and $x_{\sigma}=y_{\sigma}, \theta_{\sigma}$ or $\rho_{\sigma}$ can be made in an univocal way when $g$ is not identically equal to zero (see Proposition 6). Particularly, $\rho_{\varepsilon}: \rho_{\sigma}$ $\in I \rightarrow I$ is a continuous function such that $\rho_{\varepsilon}(1)=1$ and

$$
\frac{\mathrm{d} \rho_{\varepsilon}}{\mathrm{d} \rho_{\sigma}}=\delta_{f}^{-3} \frac{\mathrm{d} f_{1}}{\mathrm{~d} \rho_{\sigma}} g f>0, \quad \forall \rho_{\sigma} \in\left[\frac{1}{2}, 1[.\right.
$$

The case where $g$ vanishes is a particular one and leads to a non-univocal correspondence

$$
\rho_{\varepsilon}=1, \quad \forall \rho_{\sigma} \in I .
$$

\section{PHASE TRANSFORMATION SURFACE EQUATION}

Under the hypothesis providing the univocal correspondence between $\boldsymbol{\sigma}$ and $\boldsymbol{\epsilon}_{\sigma}$, one can write the equation of the phase transformation surface as in (35)

$$
\mathcal{H}\left(\boldsymbol{\epsilon}_{\sigma}\right) \equiv \overline{\boldsymbol{\epsilon}}_{\sigma} h\left(x_{\varepsilon}\right)=1,
$$

where

$$
h\left(x_{\varepsilon}\right) \equiv \frac{\kappa^{-2}}{\delta_{f}\left(x_{\sigma}\right)},
$$


$x_{\varepsilon}$ and $x_{\sigma}$ being linked in an univocal way via (38).

The eigenvalues $\lambda_{k}^{\epsilon}$ of $\epsilon_{\sigma}$ (see (14)) can be written as

$$
\left\{\begin{array}{l}
\lambda_{1}^{\epsilon}=\rho_{1}^{\epsilon} \equiv \delta_{f}\left(x_{\sigma}\right)\left(-\frac{1}{2} \rho_{\epsilon}-\frac{\sqrt{3}}{2}\left(1-\rho_{\epsilon}^{2}\right)^{\frac{1}{2}}\right)=\delta_{f}\left(x_{\sigma}\right) \cos \left(\theta_{\sigma}-\chi_{\sigma}+\frac{2 \pi}{3}\right) \\
\lambda_{2}^{\epsilon}=\rho_{2}^{\epsilon} \equiv \delta_{f}\left(x_{\sigma}\right)\left(-\frac{1}{2} \rho_{\epsilon}+\frac{\sqrt{3}}{2}\left(1-\rho_{\epsilon}^{2}\right)^{\frac{1}{2}}\right)=\delta_{f}\left(x_{\sigma}\right) \cos \left(\theta_{\sigma}-\chi_{\sigma}+\frac{4 \pi}{3}\right) \\
\lambda_{3}^{\epsilon}=\rho_{3}^{\epsilon} \equiv \delta_{f}\left(x_{\sigma}\right) \rho_{\epsilon}=\delta_{f}\left(x_{\sigma}\right) \cos \left(\theta_{\sigma}-\chi_{\sigma}\right) .
\end{array}\right.
$$

\subsection{Reverse Transformation}

One verifies (see Proposition 8) that

$$
\chi_{\sigma}+\chi_{\epsilon}=0
$$

where $\left.\chi_{\epsilon} \in\right]-\frac{\pi}{2}, \frac{\pi}{2}\left[\right.$, is given just as for $\chi_{\sigma}$ by

$$
\chi_{\epsilon}=\arctan \left(a\left(x_{\varepsilon}\right) \frac{h^{\prime}\left(x_{\varepsilon}\right)}{h\left(x_{\varepsilon}\right)}\right) \text {. }
$$

One can also write

$$
a\left(x_{\varepsilon}\right) \frac{h^{\prime}\left(x_{\varepsilon}\right)}{h\left(x_{\varepsilon}\right)}+a\left(x_{\sigma}\right) \frac{f^{\prime}\left(y_{\sigma}\right)}{f\left(y_{\sigma}\right)}=0,
$$

or in a more explicit way (see (18))

$$
\left(1-\rho_{\epsilon}^{2}\right)^{\frac{1}{2}} \frac{\frac{\mathrm{d} h}{\mathrm{~d} \rho_{\epsilon}}}{h}+\left(1-\rho_{\sigma}^{2}\right)^{\frac{1}{2}} \frac{\frac{\mathrm{d} f}{\mathrm{~d} \rho_{\sigma}}}{f}=0 .
$$

Particularly, for $x_{\sigma}=y_{\sigma}$, one obtains

$$
\left(1-y_{\varepsilon}^{2}\right)^{\frac{1}{2}} \frac{h^{\prime}\left(y_{\varepsilon}\right)}{h\left(y_{\varepsilon}\right)}+\left(1-y_{\sigma}^{2}\right)^{\frac{1}{2}} \frac{f^{\prime}\left(y_{\sigma}\right)}{f\left(y_{\sigma}\right)}=0 .
$$

In a general way, by using (42), one deduces the inverse application:

$$
\left\{\begin{array}{l}
\theta_{\sigma}=\theta_{\varepsilon}+\chi_{\epsilon} \\
\rho_{\sigma}=\cos \left(\arccos \rho_{\epsilon}+\chi_{\epsilon}\right) \\
y_{\sigma}=\cos \left(\arccos y_{\epsilon}+3 \chi_{\epsilon}\right) .
\end{array}\right.
$$




\subsection{Duality}

We are now ready to state a result of duality type. Just as for $\mathcal{F}$, the derivative of $\mathcal{H}$ with respect to $\epsilon_{\sigma}$, see (16), takes the form

$$
\mathcal{H}^{\prime}\left(\boldsymbol{\epsilon}_{\sigma}\right)=h\left(x_{\epsilon}\right) \mathbf{n}_{\sigma}+a\left(x_{\epsilon}\right) h^{\prime}\left(x_{\epsilon}\right) \mathbf{n}_{\sigma}^{\perp}
$$

where

$$
\left\{\begin{array}{l}
\mathbf{n}_{\sigma}=\rho_{\epsilon} \mathbf{e}_{1}^{\mathbf{R}_{\sigma}}-\left(1-\rho_{\epsilon}^{2}\right)^{\frac{1}{2}} \mathbf{e}_{2}^{\mathbf{R}_{\sigma}} \\
\mathbf{n}_{\sigma}^{\perp} \equiv\left(1-\rho_{\epsilon}^{2}\right)^{\frac{1}{2}} \mathbf{e}_{1}^{\mathbf{R}_{\sigma}}+\rho_{\epsilon} \mathbf{e}_{2}^{\mathbf{R}_{\sigma}} .
\end{array}\right.
$$

We show (see Proposition 9) that

$$
\mathcal{H}^{\prime}\left(\boldsymbol{\epsilon}_{\sigma}\right)=\frac{1}{\sigma_{c}} \mathbf{S}_{\sigma}=\mathbf{S}_{\frac{1}{\sigma_{c}} \sigma}
$$

for all $\boldsymbol{\sigma}$ which are solutions of the yield phase transformation surface in the stress space, see (1).

In fact, it is proved that the space of yield surface of phase transformation initiation (i.e. the elastic domain in the stress space of the mother phase: the austenite) is dual to the space occupied by the domain associated to the effective transformation strain of the produced phase: the martensite. The functions $f\left(x_{\sigma}\right)$ and $h\left(x_{\epsilon}\right)$ inherit the same properties, notably that of the convexity.

The parameter $x_{\epsilon}$ can be obtained starting from $x_{\sigma}(42)$ and

$$
\chi_{\sigma}=\arctan \left(\frac{a\left(x_{\sigma}\right) f^{\prime}\left(x_{\sigma}\right)}{f\left(x_{\sigma}\right)}\right) .
$$

Reversely, the parameter $x_{\sigma}$ can be obtained from $x_{\epsilon}(49)$ and

$$
\chi_{\epsilon}=\arctan \left(a\left(x_{\varepsilon}\right) \frac{h^{\prime}\left(x_{\varepsilon}\right)}{h\left(x_{\varepsilon}\right)}\right) .
$$

The transport from one space to the another can be made in a very simple way by using the relation

$$
\chi_{\sigma}+\chi_{\epsilon}=0 \text {. }
$$

The variables $\epsilon_{\sigma}$ and $\mathbf{S}_{\sigma}$ can be considered as dual with the normality rules associated to their yield surfaces respectively.

In the next section, we give a few applications of the surface determination concerning two different shape memory alloys. 


\section{APPLICATIONS}

We consider two experimental series concerning $\mathrm{Cu}-\mathrm{Al}-\mathrm{Be}$ and NiTi alloys, whose yield values are given (in $\mathrm{MPa}$ ) in the tables below.

Concerning the $\mathrm{Cu}-\mathrm{Al}-\mathrm{Be}$ alloy, the yield points are obtained by bi-compression tests on cubes and tension (compression)- internal pressure on tubes (see Bouvet et al [9]).

$$
\mathrm{Cu}-\mathrm{Al}-\mathrm{Be}: \boldsymbol{\sigma}_{i}=\left(\begin{array}{lll}
\sigma_{1} & 0 & 0 \\
0 & \sigma_{2} & 0 \\
0 & 0 & 0
\end{array}\right), 1 \leq i \leq n=11,
$$

\begin{tabular}{lccccccccccc}
\hline$i$ & 1 & 2 & 3 & 4 & 5 & 6 & 7 & 8 & 9 & 10 & 11 \\
\hline$\sigma_{1}$ & 87.14 & 91.43 & 105.71 & 45.71 & 0 & -45.71 & -105.71 & -100 & -65.71 & 0 & 51.43 \\
$\sigma_{2}$ & 0 & 42.86 & 102.86 & 100 & 80 & 60 & 0 & -48.57 & -91.43 & -100 & -48.57 \\
\hline
\end{tabular}

As for the Ni-Ti alloy, the tension (compression)-torsion tests on tubes as performed by Laverhne-Taillard et al. [10], give the following yield values:

$$
\text { NiTi: } \boldsymbol{\sigma}_{i}=\left(\begin{array}{ccc}
0 & 0 & 0 \\
0 & 0 & \sigma_{z \theta} \\
0 & \sigma_{z \theta} & \sigma_{z z}
\end{array}\right), 1 \leq i \leq n=15
$$

\begin{tabular}{lccccccccccccccc}
\hline$i$ & 1 & 2 & 3 & 4 & 5 & 6 & 7 & 8 & 9 & 10 & 11 & 12 & 13 & 14 & 15 \\
\hline$\sigma_{z z}$ & 400 & 390 & 310 & 220 & 0 & -210 & -370 & -520 & -530 & -520 & -430 & -200 & 30 & 250 & 370 \\
$\sigma_{z \theta}$ & 0 & 150 & 240 & 340 & 440 & 420 & 330 & 220 & 0 & -150 & -340 & -400 & -370 & -300 & -150 \\
\hline
\end{tabular}

\subsection{Shape Function Choice}

We will use the shape function $f$ which has been introduced in [9], namely

$$
f(y)=\cos \left(\frac{1}{3} \arccos (1-a(1-y))\right) .
$$

We show (see Proposition 7) that $\mathcal{F}$ is convex if and only if

$$
0 \leq a \leq 1
$$

We obtain the following normalization constant $\sigma_{c}$ and the parameter $a$ of the function $f$ (see Figure 3):

$$
\mathrm{Cu}-\mathrm{Al}-\mathrm{Be}:\left\{\begin{array} { r } 
{ a = 0 . 4 7 } \\
{ \sigma _ { c } = 8 9 . 6 1 }
\end{array} \text { and NiTi : } \left\{\begin{array}{rl}
a & =0.63 \\
\sigma_{c} & =424.31
\end{array}\right.\right.
$$




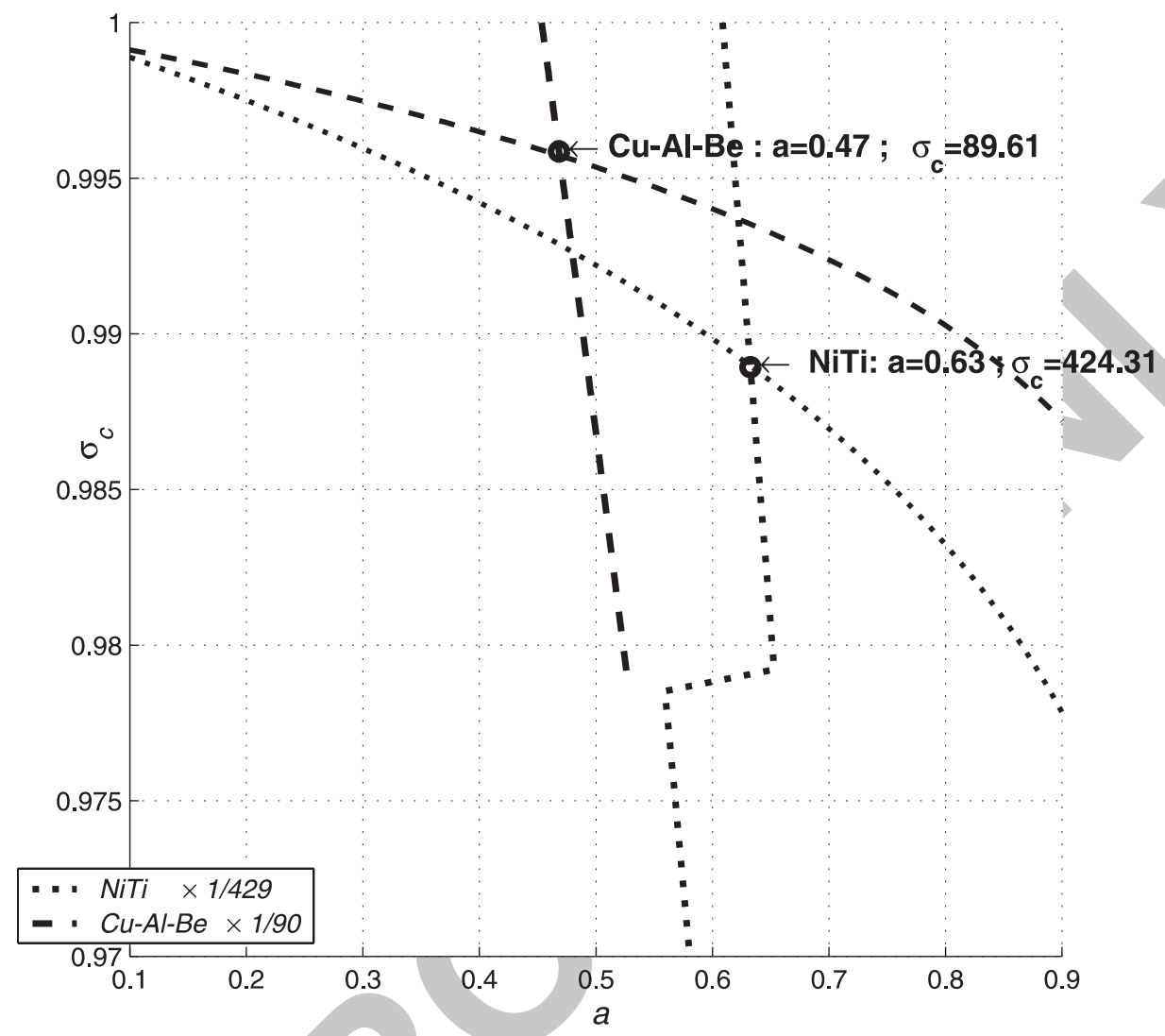

Figure 3. Obtention of $a$ and $\sigma_{c}$ parameters.

They are determined by the intersection of the two curves $\sigma_{c}(a)$ and the "reverse" formulae $a\left(\sigma_{c}\right)$ with the following definitions:

$$
\sigma_{c}(a) \equiv \frac{\sum_{i=1}^{n} \overline{\boldsymbol{\sigma}}_{i} \cos \left(\frac{1}{3} \arccos \left(1-a\left(1-y_{\sigma_{i}}\right)\right)\right)}{\sum_{i=1}^{n} \cos \left(\frac{1}{3} \arccos \left(1-a\left(1-y_{\sigma_{i}}\right)\right)\right)},
$$

and

$$
a\left(\sigma_{c}\right) \equiv \frac{\sum_{\substack{i=1 \\ \frac{\sigma_{\bar{c}} \leq 1}{\bar{\sigma}_{i}}}}^{n}\left(1-\cos \left(3 \arccos \left(\frac{\sigma_{c}}{\overline{\boldsymbol{\sigma}}_{i}}\right)\right)\right)}{\sum_{\substack{i=1 \\ \bar{\sigma}_{c} \leq 1}}^{n}\left(1-y_{\sigma_{i}}\right)}
$$




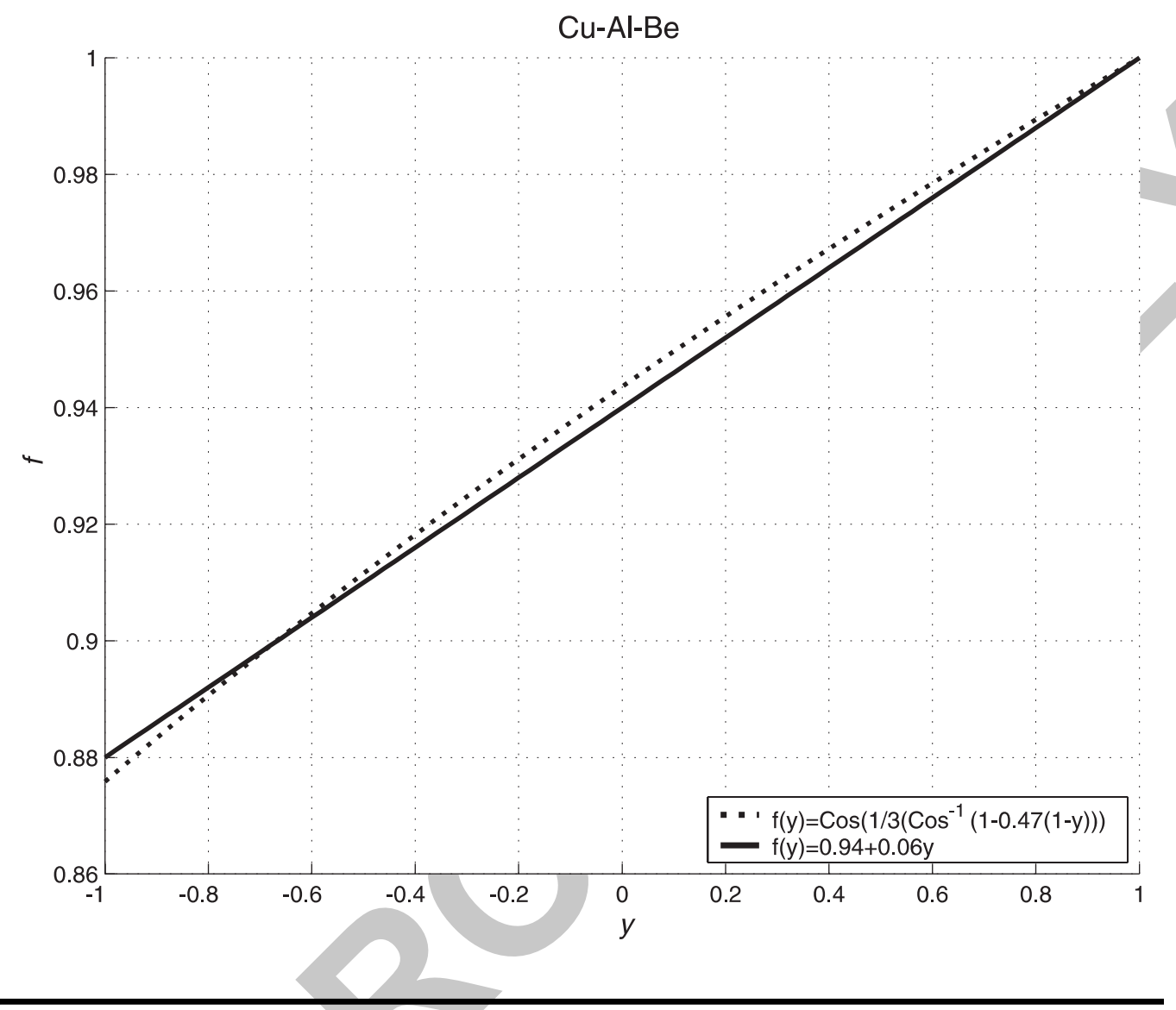

Figure 4. Comparison between "cosinus" formulation and straight lines for Cu-Al-Be alloy.

In fact, the function defined in (53) behaves as $f(\rho)=1-\alpha+\alpha \rho^{q}$ where $\alpha$ and $q$ are given by the formulae (86) and (87) (see Appendix A)

$$
\mathrm{Cu}-\mathrm{Al}-\mathrm{Be}:\left\{\begin{array} { l } 
{ \alpha = 0 . 1 3 } \\
{ q = 4 . 5 3 }
\end{array} \text { and NiTi : } \left\{\begin{array}{l}
\alpha=0.19 \\
q=4.20
\end{array}\right.\right.
$$

or more simply as the classical linear function

$$
f(y)=b_{1}+b_{2} y,
$$

with the following parameters:

$$
\mathrm{Cu}-\mathrm{Al}-\mathrm{Be}:\left\{\begin{array}{l}
b_{1}=0.94 \\
b_{2}=0.06
\end{array} \text { and NiTi }:\left\{\begin{array}{l}
b_{1}=0.91 \\
b_{2}=0.09
\end{array}\right.\right.
$$

Figures 4 and 5 show the comparison of the "cosinus" formulation with the linear form for the above parameters which are obtained by minimizing the functional 


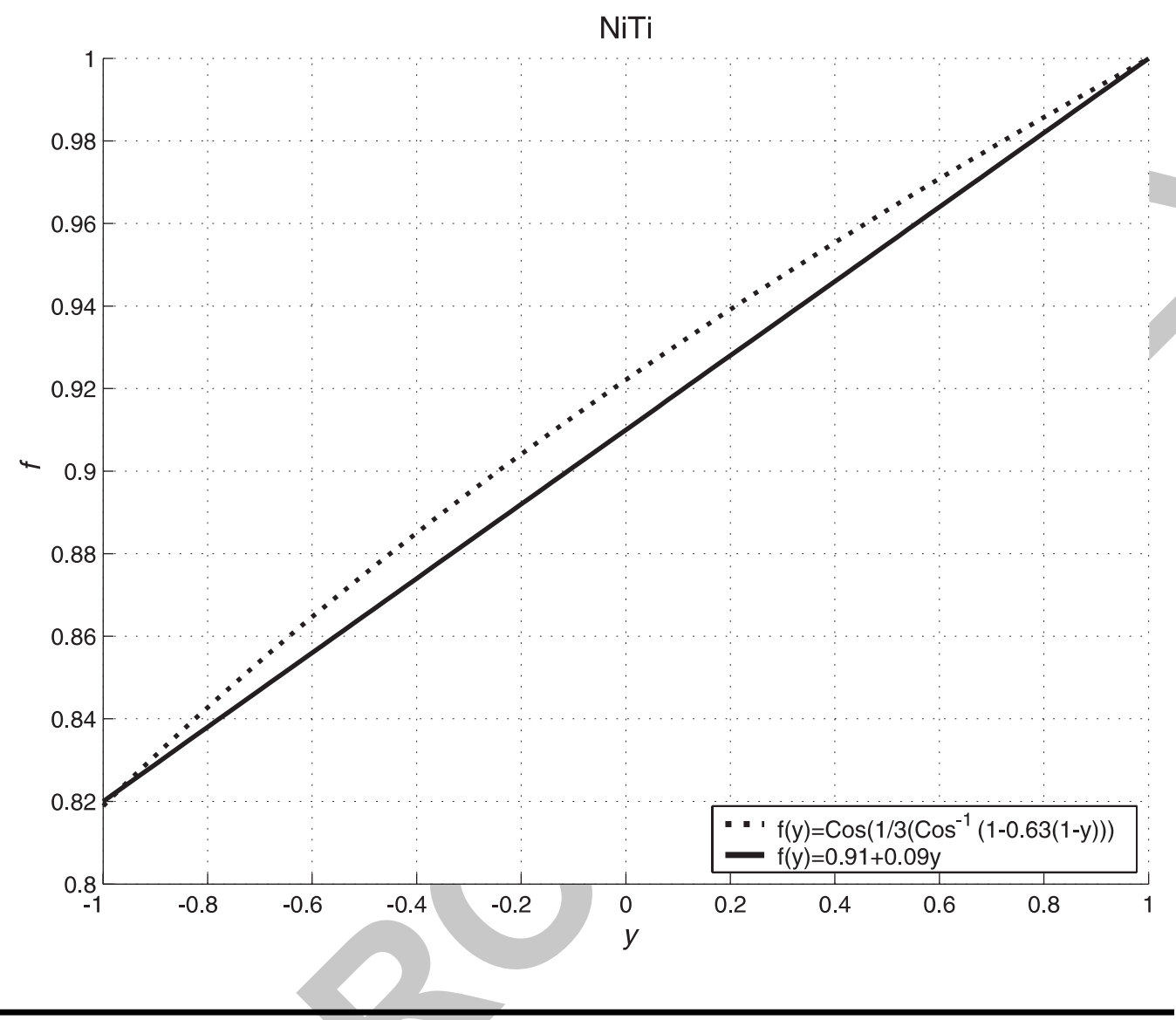

Figure 5. Comparison between "cosinus" formulation and straight lines for $\mathrm{Ni}-\mathrm{Ti}$ alloy.

$$
J\left(b_{1}, b_{2}\right) \equiv\left(\sum_{i=1}^{n}\left|\mathcal{F}(\boldsymbol{\sigma})-\sigma_{c}\right|^{2}\right)^{\frac{1}{2}}
$$

In any case the convexity condition is satisfied (apply Proposition 10 with $b=\frac{b_{2}}{b_{1}}=$ $\left.\frac{0.09}{0.91}=9.8901 \times 10^{-2}\right)$.

\subsection{Application 1: $\mathrm{Cu}-\mathrm{Al}-\mathrm{Be}$}

The normalized solutions of (1) given by (15) are characterized by the couples

$$
\left(\sigma_{1}, \sigma_{2}\right) \in\left\{\frac{1}{3}\left(\rho_{j}+2 \rho_{i}, \rho_{i}+2 \rho_{j}\right) \mid \forall \rho \in I ; \quad 1 \leq i \neq j \leq 3\right\},
$$

and those given by (44) by 
Cu-Al-Be : $\sigma_{c}=89.61$

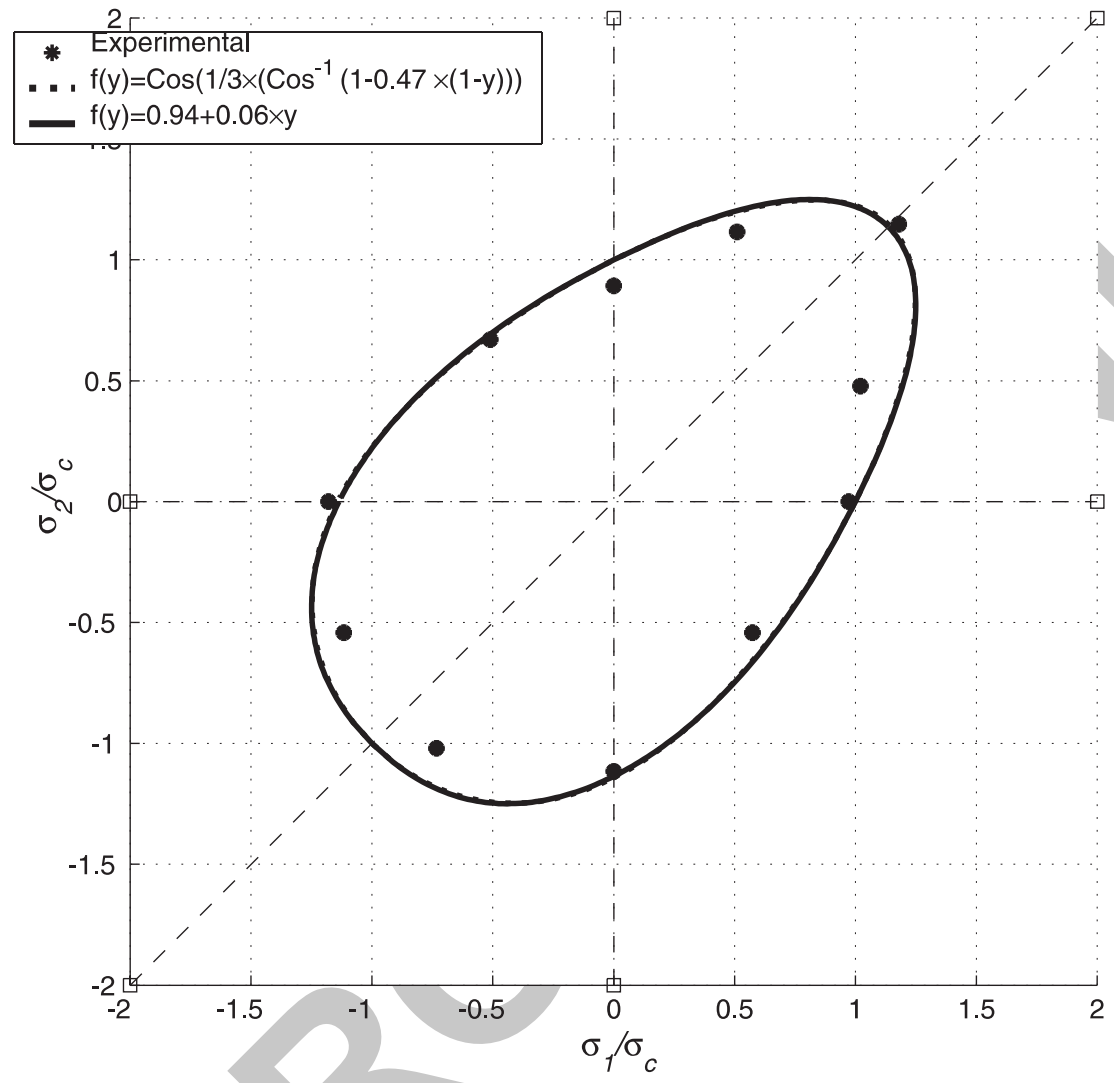

Figure 6. Yield surfaces in the stress space for Cu-Al-Be alloy.

$$
\left(\epsilon_{1}, \epsilon_{2}\right) \in\left\{\left(\rho_{i}^{\epsilon}, \rho_{j}^{\epsilon}\right) \mid \forall \rho \in I ; \quad 1 \leq i \neq j \leq 3\right\},
$$

see Figures 6-8.

\subsection{Application 2: NiTi}

In this case, the eigenvalues of $\boldsymbol{\sigma}$ are

$$
\lambda_{3}=\frac{1}{2}\left(\sigma_{z z}+\sqrt{\sigma_{z z}^{2}+4 \sigma_{z \theta}^{2}}\right), \quad \lambda_{2}=0 \quad \text { and } \quad \lambda_{1}=\frac{1}{2}\left(\sigma_{z z}-\sqrt{\sigma_{z z}^{2}+4 \sigma_{z \theta}^{2}}\right) .
$$

The normalized solutions of (1) given by (15) are such that

$$
\sigma_{z z}=\lambda_{1}+\lambda_{3} ; \quad \sigma_{z \theta}= \pm \sqrt{-\lambda_{1} \lambda_{3}},
$$




\section{Cu-Al-Be : $\sigma_{c}=89.61$}

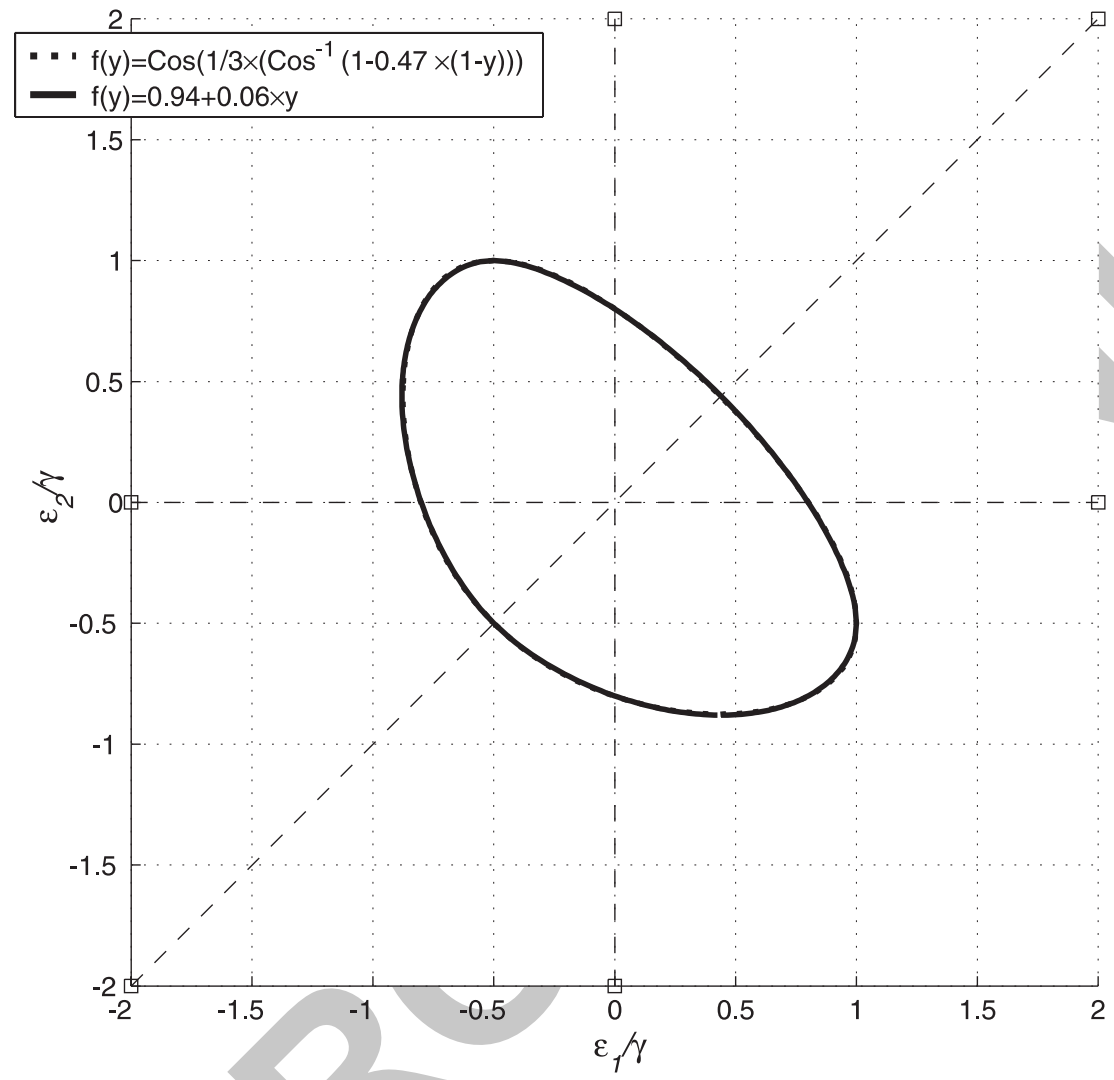

Figure 7. Yield surfaces in the phase transformation strain dual space for $\mathrm{Cu}-\mathrm{Al}-\mathrm{Be}$ alloy.

where

$$
\left(\lambda_{1}, \lambda_{3}\right)=\frac{1}{3}\left(2 \rho_{3}+\rho_{1}, 2 \rho_{1}+\rho_{3}\right)
$$

i.e.

$$
\sigma_{z z}=-\rho_{2} ; \quad \sigma_{z \theta}= \pm \frac{1}{3} \sqrt{\left(\rho_{3}-\rho_{2}\right)\left(\rho_{2}-\rho_{1}\right)}
$$

As for the solutions given by (44), they are characterized by the couples

$$
\epsilon_{z z}=-\rho_{2}^{\epsilon} ; \quad \epsilon_{z \theta}= \pm \frac{1}{3} \sqrt{\left(\rho_{3}^{\epsilon}-\rho_{2}^{\epsilon}\right)\left(\rho_{2}^{\epsilon}-\rho_{1}^{\epsilon}\right)}
$$

see Figures 9-11. 


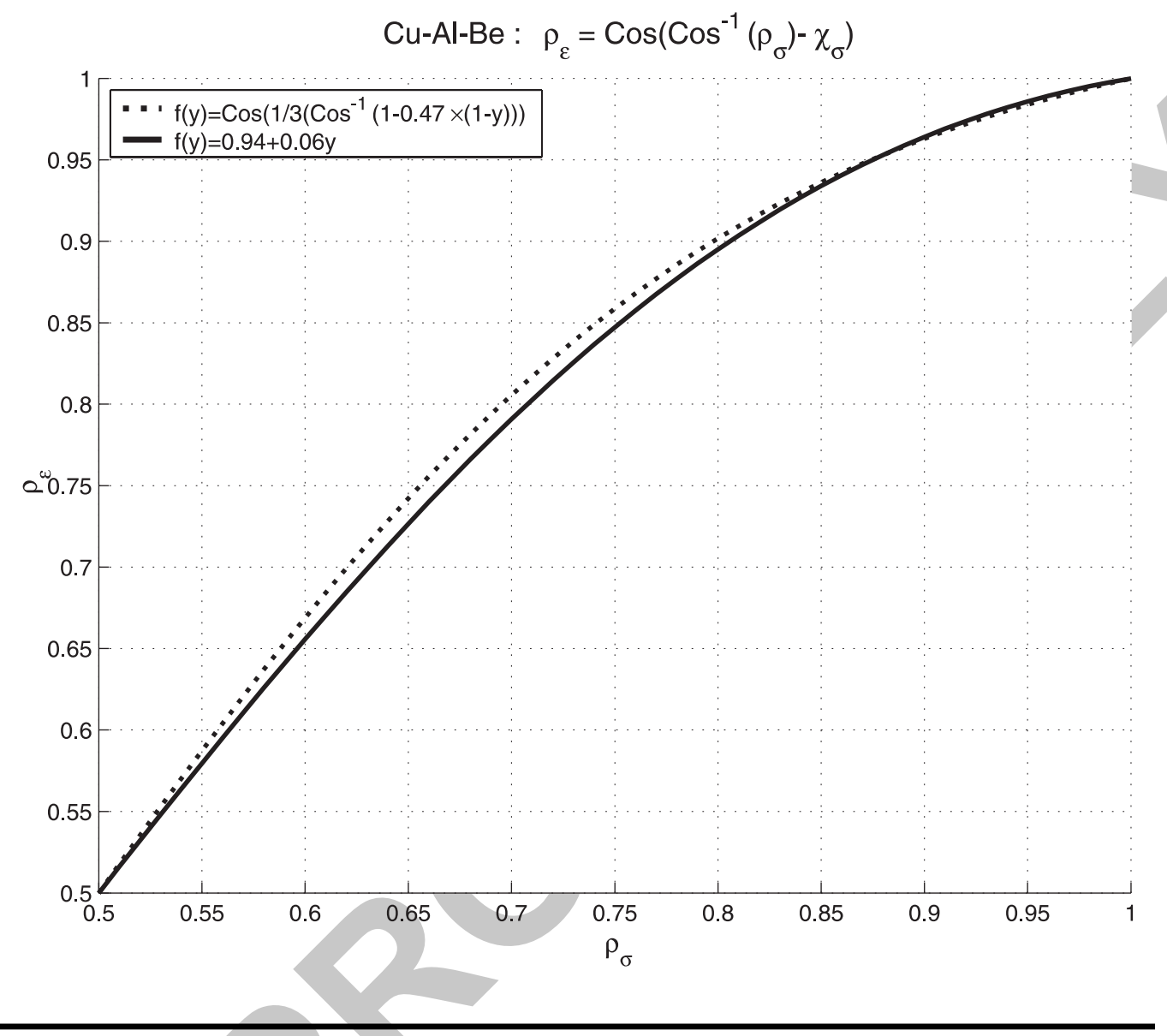

Figure 8. Univocal correspondence between $\rho_{\sigma}$ and $\rho_{\epsilon}$ for Cu-Al-Be alloy.

\section{CONCLUSION}

We have performed the modelling of the yield curves of phase transformation initiation for isotropic SMA. materials, in the stress space, by taking into account the asymmetry between tension and compression. Many choices of the shape function $f(y)$ are possible notably a linear expression, one with a "cosinus" formulation or a power law in terms of $\rho$, and in every case, the fitting of experimental results are good. We give a necessary and sufficient condition for convexity of the yield curve thus completing the necessary one as stated by Raniecki and Mroz [3] and Bigoni and Piccolroaz [7].

Special attention is paid to the transport of these yield surfaces from the stress space to the one of effective transformation strain of the produced phase (i.e. the martensite). A duality is established between these two surfaces. An extension of the investigation is possible concerning anisotropic shape memory alloys as drawn or rolled SMA bars. 
$\mathrm{NiTi}: \sigma_{c}=421.31$

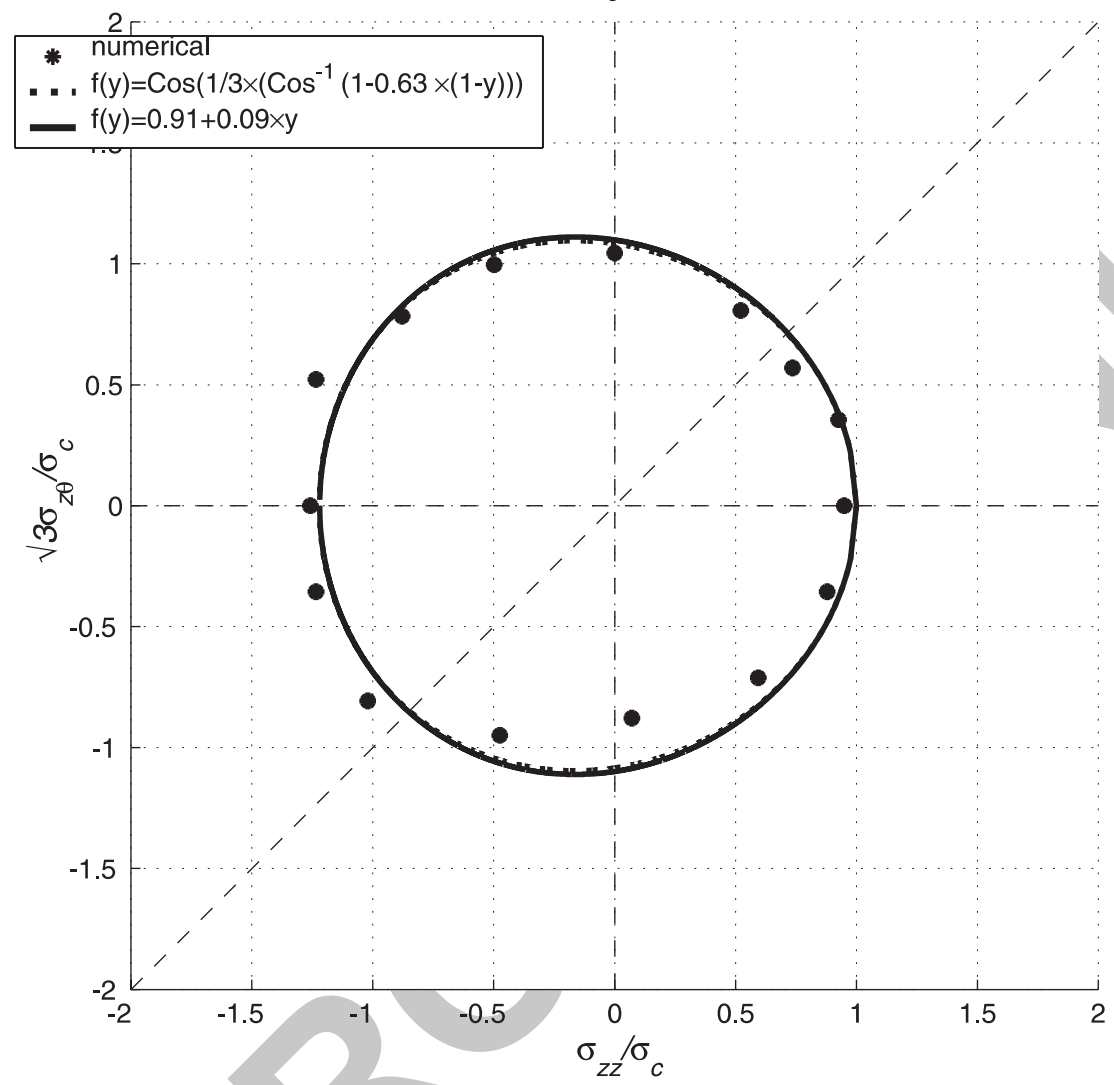

Figure 9. Yield surfaces in the stress space for $\mathrm{Ni}-\mathrm{Ti}$ alloy.

\section{PROOFS OF THE PROPOSITIONS}

Proposition 1. For any choice of $x\left(y_{\sigma}, \theta_{\sigma}\right.$ or $\left.\rho_{\sigma}\right), \mathbf{N}_{\sigma}$ can be written as

$$
\mathbf{N}_{\sigma}=\rho(x) \mathbf{e}_{1}^{\mathbf{R}_{\sigma}}-\left(1-|\rho(x)|^{2}\right)^{\frac{1}{2}} \mathbf{e}_{2}^{\mathbf{R}_{\sigma}}
$$

Moreover, $\rho$ is equal to

$$
\rho=\kappa^{-2}\left(\mathbf{N}_{\sigma}: \mathbf{e}_{1}^{\mathbf{R}_{\sigma}}\right)
$$

Proof. The eigenvalues $\lambda_{k}$ of $\mathbf{N}_{\sigma}$ solve

$$
\lambda^{3}-\frac{3}{4} \lambda-\frac{1}{4} y_{\sigma}=0 .
$$




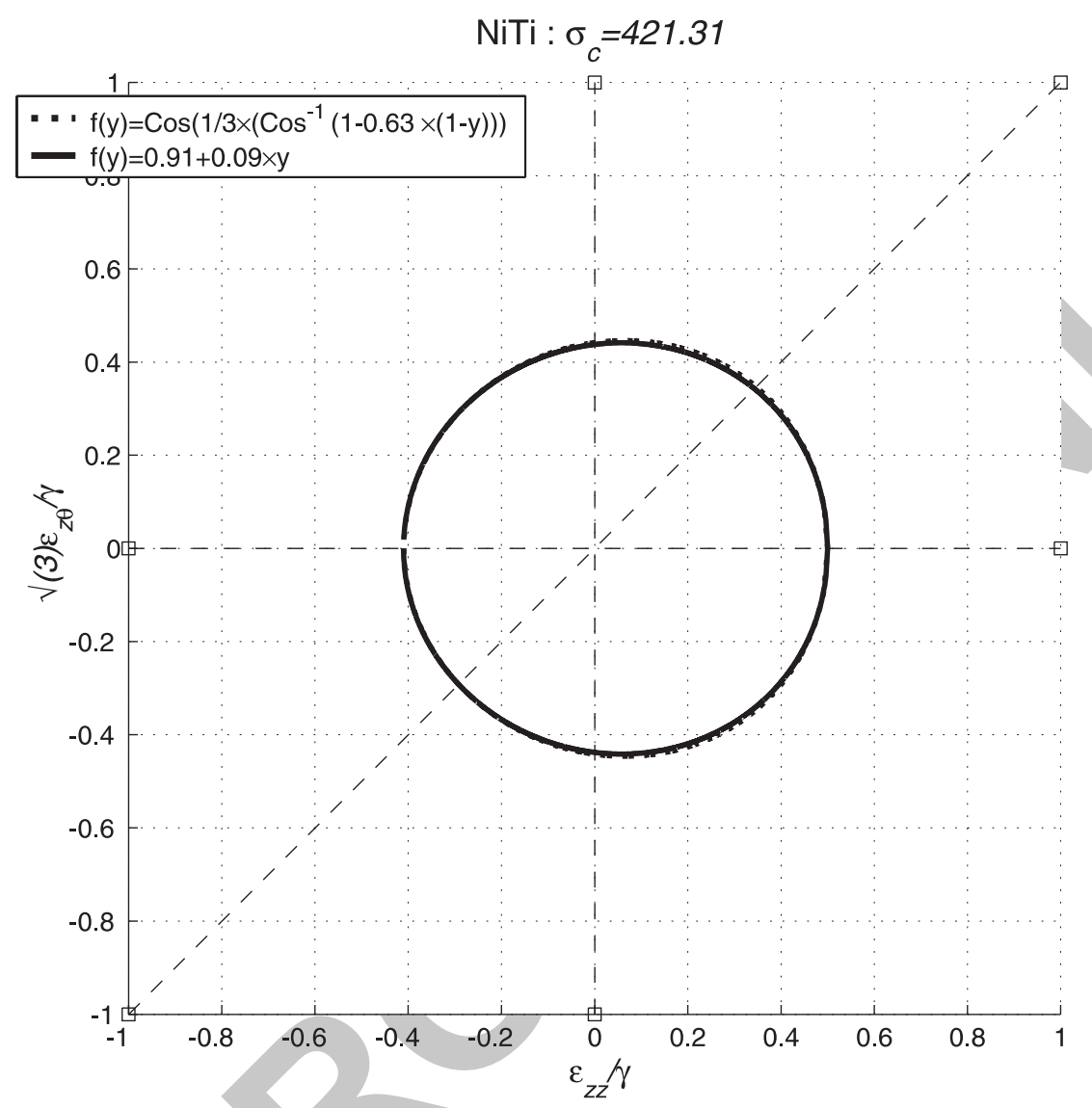

Figure 10. Yield surfaces in the phase transformation strain dual space for $\mathrm{Ni}$ - $\mathrm{Ti}$ alloy.

One can verify that $y_{\sigma} \equiv 4 \operatorname{det}\left(\mathbf{N}_{\sigma}\right) \in[-1,1]$ and that the Cardan discriminant is equal to

$$
\Delta=-\frac{1}{4^{2}}\left(1-y_{\sigma}^{2}\right)
$$

If $\Delta=0$, the equation has three real roots: one single and one double. One has

$$
y_{\sigma}=1 \Rightarrow \lambda_{1}=\lambda_{2}=-\frac{1}{2} \quad \text { and } \quad \rho=\lambda_{3}=1
$$

or

$$
y_{\sigma}=-1 \Rightarrow \lambda_{1}=-1 \quad \text { and } \quad \rho=\lambda_{2}=\lambda_{3}=\frac{1}{2}
$$

In the general case $\Delta<0$, the equation has three real distinct roots given by 


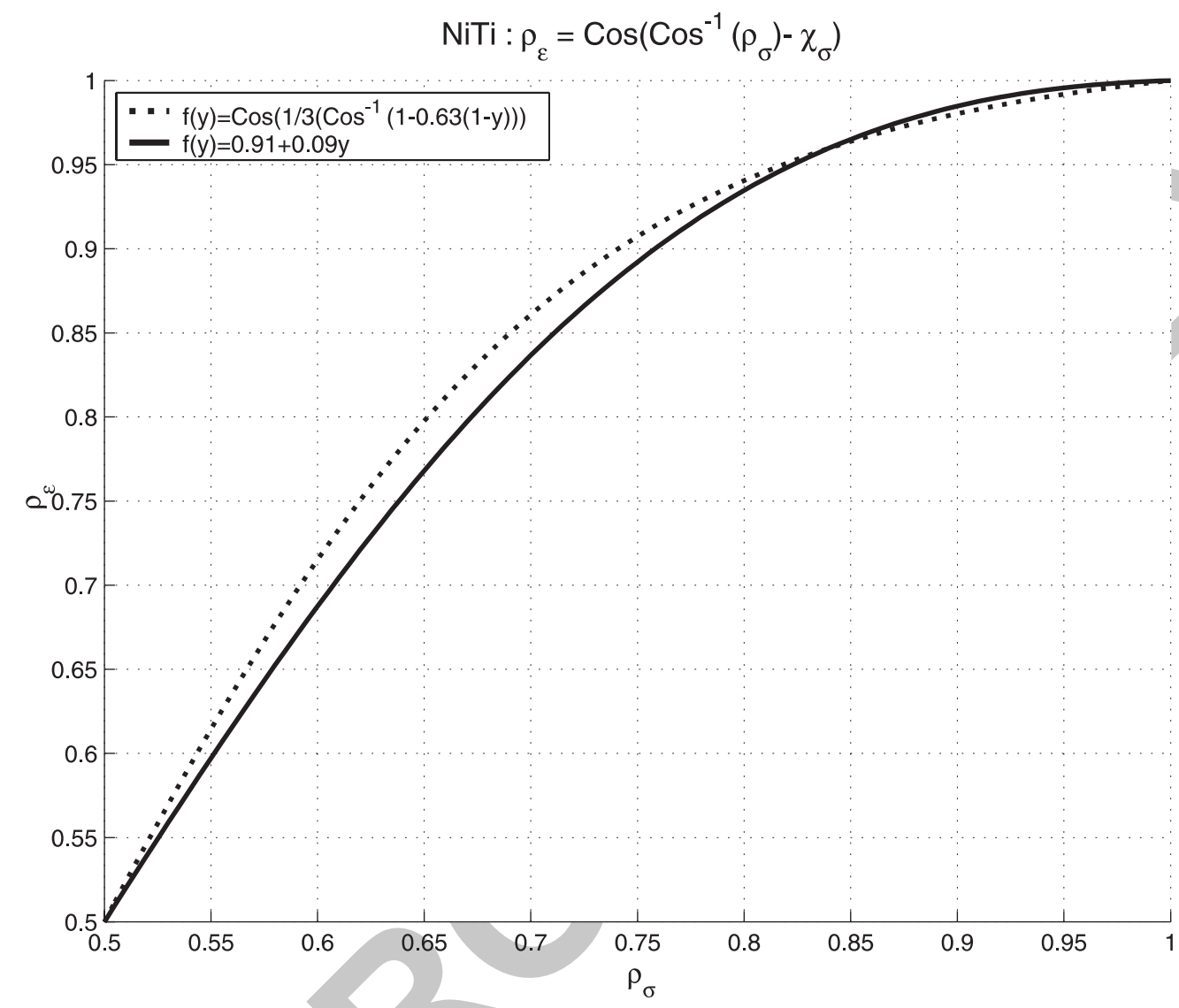

Figure 11. Univocal correspondence between $\rho_{\sigma}$ and $\rho_{\epsilon}$ for $\mathrm{Ni}$-Ti alloy.

$$
\lambda_{k}=\cos \left(\frac{\arccos \left(y_{\sigma}\right)+2 k \pi}{3}\right), \quad k=1,2,3,
$$

i.e.

$$
\lambda_{k}=\cos \left(\frac{1}{3} \arccos \left(y_{\sigma}\right)\right) \cos \left(\frac{2 k}{3} \pi\right)-\sin \left(\frac{1}{3} \arccos \left(y_{\sigma}\right)\right) \sin \left(\frac{2 k}{3} \pi\right) .
$$

So, the diagonal matrix composed of the eigenvalues of $\mathbf{N}_{\sigma}$ can be written as

$$
\widetilde{\Lambda}_{\sigma}=\rho_{\sigma} \mathbf{e}_{1}-\left(1-\rho_{\sigma}^{2}\right)^{\frac{1}{2}} \mathbf{e}_{2},
$$

where $\rho_{\sigma}=\cos \left(\frac{1}{3} \arccos \left(y_{\sigma}\right)\right), \mathbf{e}_{1}=\operatorname{diag}\left(\cos \left(\frac{2 k}{3} \pi\right)\right)$ and $\mathbf{e}_{2}=\operatorname{diag}\left(\sin \left(\frac{2 k}{3} \pi\right)\right)$. $\mathbf{e}_{2}^{\mathbf{R}_{\sigma}}$.

The expression of $\rho_{\sigma}$ in (56) is obtained by the orthogonality (13) between $\mathbf{e}_{1}^{\mathbf{R}_{\sigma}}$ and 
Proposition 2. Let $a(x)$ and $\mathbf{N}_{\sigma}^{\perp}$ defined by (18) and (17). The derivative of $\mathcal{F}$ is given by (16)

$$
\frac{\partial \mathcal{F}}{\partial \boldsymbol{\sigma}}=f(x) \mathbf{N}_{\sigma}+a(x) f^{\prime}(x) \mathbf{N}_{\sigma}^{\perp}
$$

Moreover

$$
\mathcal{F}^{\prime}(\boldsymbol{\sigma}): \boldsymbol{\sigma}=\mathcal{F}(\boldsymbol{\sigma}) \quad \forall \boldsymbol{\sigma}
$$

Proof. We are going to use the following equation of the derivative of $\mathcal{F}$

$$
\frac{\partial \mathcal{F}}{\partial \boldsymbol{\sigma}}=f(x) \frac{\partial \overline{\boldsymbol{\sigma}}}{\partial \boldsymbol{\sigma}}+f^{\prime}(x) \overline{\boldsymbol{\sigma}} \frac{\partial x}{\partial \boldsymbol{\sigma}}
$$

and the following identity: $\frac{\partial}{\partial \boldsymbol{\sigma}}=\frac{\partial}{\partial \mathbf{S}_{\sigma}}-\frac{1}{3} \operatorname{tr}\left(\frac{\partial}{\partial \mathbf{S}_{\sigma}}\right) \mathbf{1}$.

The first term in (61) is classical:

$$
\frac{\partial \overline{\boldsymbol{\sigma}}}{\partial \boldsymbol{\sigma}}=\kappa \frac{\partial}{\partial \boldsymbol{\sigma}}\left(\left|\mathbf{S}_{\sigma}\right|\right)=\kappa \frac{\partial}{\partial \mathbf{S}_{\sigma}}\left(\left|\mathbf{S}_{\sigma}\right|\right)=\kappa^{2} \frac{\mathbf{S}_{\sigma}}{\kappa\left|\mathbf{S}_{\sigma}\right|}=\kappa^{2} \frac{\mathbf{S}_{\sigma}}{\overline{\boldsymbol{\sigma}}}=\mathbf{N}_{\sigma}
$$

The second term is based on the characterization of $\rho$ see (56):

$$
\rho=\overline{\boldsymbol{\sigma}}^{-1}\left(\mathbf{S}_{\sigma}: \mathbf{e}_{1}^{\mathbf{R}_{\sigma}}\right) .
$$

One obtains

$$
\begin{aligned}
\frac{\partial \rho}{\partial \boldsymbol{\sigma}} & =\overline{\boldsymbol{\sigma}}^{-1} \mathbf{e}_{1}^{\mathbf{R}_{\sigma}}-\overline{\boldsymbol{\sigma}}^{-2} \frac{\partial \overline{\boldsymbol{\sigma}}}{\partial \boldsymbol{\sigma}}\left(\mathbf{S}_{\sigma}: \mathbf{e}_{1}^{\mathbf{R}_{\sigma}}\right)=\overline{\boldsymbol{\sigma}}^{-1}\left(\mathbf{e}_{1}^{\mathbf{R}_{\sigma}}-\rho \mathbf{N}_{\sigma}\right) \\
\overline{\boldsymbol{\sigma}} \frac{\partial \rho}{\partial \boldsymbol{\sigma}} & =\mathbf{e}_{1}^{\mathbf{R}_{\sigma}}-\rho\left(\rho \mathbf{e}_{1}^{\mathbf{R}_{\sigma}}-\left(1-\rho^{2}\right)^{\frac{1}{2}} \mathbf{e}_{2}^{\mathbf{R}_{\sigma}}\right)
\end{aligned}
$$

i.e.

$$
\overline{\boldsymbol{\sigma}} \frac{\mathrm{d} \rho}{\mathrm{d} x} \frac{\partial x}{\partial \boldsymbol{\sigma}}=\left(1-\rho^{2}\right)^{\frac{1}{2}}\left(\left(1-\rho^{2}\right)^{\frac{1}{2}} \mathbf{e}_{1}^{\mathbf{R}_{\sigma}}+\rho \mathbf{e}_{2}^{\mathbf{R}_{\sigma}}\right)=\left(1-\rho^{2}\right)^{\frac{1}{2}} \mathbf{N}_{\sigma}^{\perp} .
$$

This leads to

$$
\overline{\boldsymbol{\sigma}} \frac{\partial x}{\partial \boldsymbol{\sigma}}=\left(1-\rho^{2}\right)^{\frac{1}{2}} \frac{\mathrm{d} x}{\mathrm{~d} \rho}=a(x) \mathbf{N}_{\sigma}^{\perp}
$$

Finally one obtains (60) by observing that

$$
\mathcal{F}^{\prime}(\boldsymbol{\sigma}): \boldsymbol{\sigma}=\mathcal{F}^{\prime}(\boldsymbol{\sigma}): \mathbf{S}_{\sigma}=\overline{\boldsymbol{\sigma}} \kappa^{-2}\left(\mathcal{F}^{\prime}(\boldsymbol{\sigma}): \mathbf{N}_{\sigma}\right)=\overline{\boldsymbol{\sigma}} \kappa^{-2} f(x)\left(\mathbf{N}_{\sigma}: \mathbf{N}_{\sigma}\right)=\mathcal{F}(\boldsymbol{\sigma})
$$


Proposition 3. The derivative of $\mathcal{F}$ is equal to

$$
\mathcal{F}^{\prime}(\boldsymbol{\sigma})=f_{1}\left(\rho_{\sigma}\right) \mathbf{e}_{1}^{\mathbf{R}_{\sigma}}-f_{2}\left(\rho_{\sigma}\right) \mathbf{e}_{2}^{\mathbf{R}_{\sigma}},
$$

with

$$
\left\{\begin{array}{l}
f_{1}(\rho)=\rho f(x)+\left(1-\rho^{2}\right)^{\frac{1}{2}} a(x) f^{\prime}(x)=\rho f(x)+\left(1-\rho^{2}\right) \frac{\mathrm{d} f}{\mathrm{~d} \rho} \\
f_{2}(\rho)=\left(1-\rho^{2}\right)^{\frac{1}{2}} f(x)-\rho a(x) f^{\prime}(x)=\left(1-\rho^{2}\right)^{\frac{1}{2}}\left(-\rho \frac{\mathrm{d} f}{\mathrm{~d} \rho}+f(x)\right) .
\end{array}\right.
$$

And the inverse system reads

$$
\left\{\begin{aligned}
a(x) f^{\prime}(x) & =\left(1-\rho^{2}\right)^{\frac{1}{2}} f_{1}(\rho)-\rho f_{2}(\rho) \\
f(x) & =\rho f_{1}(\rho)+\left(1-\rho^{2}\right)^{\frac{1}{2}} f_{2}(\rho) .
\end{aligned}\right.
$$

Proof. By substituting $\mathbf{N}_{\sigma}$ and $\mathbf{N}_{\sigma}^{\perp}$ in (59) as a function of $\mathbf{e}_{1}^{\mathbf{R}_{\sigma}}$ and $\mathbf{e}_{2}^{\mathbf{R}_{\sigma}}$, see (9) and (17), one obtains (64) with

$$
\left\{\begin{array}{l}
f_{1}(\rho)=\rho f(x)+\left(1-\rho^{2}\right)^{\frac{1}{2}} a(x) f^{\prime}(x) \\
f_{2}(\rho)=\left(1-\rho^{2}\right)^{\frac{1}{2}} f(x)-\rho a(x) f^{\prime}(x) .
\end{array}\right.
$$

The final expression is obtained by substitution of $a(x)\left(1-\rho^{2}\right)^{-\frac{1}{2}}$ by $\frac{\mathrm{d} x}{\mathrm{~d} \rho}$ and $f^{\prime}(x)$ by $\frac{\mathrm{d} \rho}{\mathrm{d} x} \frac{\mathrm{d} f}{\mathrm{~d} \rho}$. System (66) is a simple inversion of the system (65).

Proposition 4. The expressions of $g$ and $\frac{\mathrm{d}^{2} f}{\mathrm{~d} \rho^{2}}$ are given by

$$
g(\rho)=\left\{\begin{array}{lrr}
f(x)-3 \rho\left(4 \rho^{2}-1\right) f^{\prime}(x) & \text { if } & x=y_{\sigma} \\
f(x)+\rho\left(1-\rho^{2}\right)^{-\frac{1}{2}} f^{\prime}(x) & \text { if } & x=\theta_{\sigma} \\
f(x)-\rho f^{\prime}(x) & \text { if } & x=\rho_{\sigma}
\end{array}\right.
$$

and

$$
\frac{\mathrm{d}^{2} f}{\mathrm{~d} \rho^{2}}(\rho)= \begin{cases}24 \rho f^{\prime}(x)+9\left(4 \rho^{2}-1\right)^{2} f^{\prime \prime}(x) & \text { if } x=y_{\sigma} \\ -\rho\left(1-\rho^{2}\right)^{-\frac{3}{2}} f^{\prime}(x)+\left(1-\rho^{2}\right)^{-1} f^{\prime \prime}(x) & \text { if } x=\theta_{\sigma} \\ f^{\prime \prime}(x) & \text { if } \quad x=\rho_{\sigma} .\end{cases}
$$


In particular, $g$ and $\frac{\mathrm{d}^{2} f}{\mathrm{~d} \rho^{2}}$ are two continuous functions on $I$ for $x=y_{\sigma}$ or $x=\rho_{\sigma}$, and just on $\left[\frac{1}{2}, 1\left[\right.\right.$ for $x=\theta_{\sigma}$.

Proof. On one hand we have

$$
g(\rho) \equiv f(x)-\rho \frac{\mathrm{d} x}{\mathrm{~d} \rho} f^{\prime}(x)
$$

and on the other

$$
\frac{\mathrm{d}^{2} f}{\mathrm{~d} \rho^{2}}=\frac{\mathrm{d}^{2} x}{\mathrm{~d} \rho^{2}} f^{\prime}(x)+\left(\frac{\mathrm{d} x}{\mathrm{~d} \rho}\right)^{2} f^{\prime \prime}(x)
$$

So by substituting

$$
\frac{\mathrm{d} x}{\mathrm{~d} \rho}=\left\{\begin{array}{ll}
3\left(4 \rho^{2}-1\right) & \text { if } x=y_{\sigma} \\
-\left(1-\rho^{2}\right)^{-\frac{1}{2}} & \text { if } x=\theta_{\sigma} \\
1 & \text { if } x=\rho_{\sigma}
\end{array} \quad \text { and } \frac{\mathrm{d}^{2} x}{\mathrm{~d} \rho^{2}}= \begin{cases}24 \rho & \text { if } x=y_{\sigma} \\
-\rho\left(1-\rho^{2}\right)^{-\frac{3}{2}} & \text { if } x=\theta_{\sigma} \\
0 & \text { if } x=\rho_{\sigma}\end{cases}\right.
$$

in the above, we finally recover the two identities (67) and (68). The continuity of the functions $g$ and $\frac{\mathrm{d}^{2} f}{\mathrm{~d} \rho^{2}}$ is trivial.

Proposition 5. Let $g$ and $\frac{\mathrm{d}^{2} f}{\mathrm{~d} \rho^{2}}$ be continuous on $I$. Then $\mathcal{F}$ is a convex function if and only if the condition (22), i.e.

$$
\left\{\begin{array}{l}
g(\rho) \geq 0 \\
\frac{\mathrm{d} g}{\mathrm{~d} \rho}(\rho) \leq 0
\end{array}\right.
$$

is verified for all $\rho \in I$.

Proof. A necessary and sufficient condition for the convexity of $\mathcal{F}$ is based on the following inequality:

$$
\mathcal{F}(\boldsymbol{\sigma}) \geq \mathcal{F}(\mathbf{b})+\left(\mathcal{F}^{\prime}(\mathbf{b}):(\boldsymbol{\sigma}-\mathbf{b})\right), \quad \forall \boldsymbol{\sigma}, \mathbf{b}
$$

With $\operatorname{tr}\left(\mathcal{F}^{\prime}(\mathbf{b})\right)=0$ and $\mathcal{F}^{\prime}(\mathbf{b}): \mathbf{b}=\mathcal{F}(\mathbf{b})$ (see (60)), the inequality is reduced to

$$
\mathcal{F}(\boldsymbol{\sigma}) \geq\left(\mathcal{F}^{\prime}(\mathbf{b}): \mathbf{S}_{\sigma}\right), \quad \forall \boldsymbol{\sigma}, \mathbf{b}
$$

or by multiplying by $\kappa^{2} \overline{\boldsymbol{\sigma}}^{-1}$,

$$
f(x) \geq \kappa^{-2}\left(\mathcal{F}^{\prime}(\mathbf{b}): \mathbf{N}_{\sigma}\right)
$$


The calculation of $\left(\mathcal{F}^{\top}(\mathbf{b}): \mathbf{N}_{\sigma}\right)$ gives

$$
\begin{aligned}
\mathcal{F}^{\prime}(\mathbf{b}): \mathbf{N}_{\sigma} & =\left(f_{1}\left(\rho_{b}\right) \mathbf{e}_{1}-f_{2}\left(\rho_{b}\right) \mathbf{e}_{2}: \rho \mathbf{e}_{1}-\left(1-\rho^{2}\right)^{\frac{1}{2}} \mathbf{e}_{2}\right) \\
& =\kappa^{2}\left(f_{1}\left(\rho_{b}\right) \rho+f_{2}\left(\rho_{b}\right)\left(1-\rho^{2}\right)^{\frac{1}{2}}\right) .
\end{aligned}
$$

The inequality (71) then becomes

$$
f(x) \geq \rho f_{1}\left(\rho_{b}\right)+\left(1-\rho^{2}\right)^{\frac{1}{2}} f_{2}\left(\rho_{b}\right) \quad \forall \rho, \rho_{b} \in I .
$$

To show (72) let us introduce the function $u(\rho)$ as

$$
u(\rho)=\rho f_{1}\left(\rho_{b}\right)+\left(1-\rho^{2}\right)^{\frac{1}{2}} f_{2}\left(\rho_{b}\right)-f(x),
$$

with $\rho_{b}$ fixed on $I$. We must prove that

$$
u(\rho) \leq 0, \quad \forall \rho \in I .
$$

Two cases will be investigated:

- Case $\rho_{b} \neq 1$. For $\rho=\rho_{b}$, one obtains (66):

$$
u\left(\rho_{b}\right)=\rho_{b} f_{1}\left(\rho_{b}\right)+\left(1-\rho_{b}^{2}\right)^{\frac{1}{2}} f_{2}\left(\rho_{b}\right)-f\left(x_{b}\right)=0 .
$$

As the first derivative of $u$ is

$$
\frac{\mathrm{d} u}{\mathrm{~d} \rho}=f_{1}\left(\rho_{b}\right)-\rho\left(1-\rho^{2}\right)^{-\frac{1}{2}} f_{2}\left(\rho_{b}\right)-\frac{\mathrm{d} f}{\mathrm{~d} \rho},
$$

by using (18), $\frac{\mathrm{d} u}{\mathrm{~d} \rho}$ can be written as

$$
\frac{\mathrm{d} u}{\mathrm{~d} \rho}=\left(1-\rho^{2}\right)^{-\frac{1}{2}}\left(\left(1-\rho^{2}\right)^{\frac{1}{2}} f_{1}\left(\rho_{b}\right)-\rho f_{2}\left(\rho_{b}\right)-a(x) f^{\prime}(x)\right) .
$$

So, for $\rho=\rho_{b}$, with $\rho_{b} \neq 1$, thanks to (66), one obtains $\frac{\mathrm{d} u}{\mathrm{~d} \rho}\left(\rho_{b}\right)=0$. A second derivation gives

$$
\frac{\mathrm{d}^{2} u}{\mathrm{~d} \rho^{2}}=-\left(1-\rho^{2}\right)^{-\frac{3}{2}} f_{2}\left(\rho_{b}\right)-\frac{\mathrm{d}^{2} f}{\mathrm{~d} \rho^{2}} .
$$

By the Taylor formula at the neighborhood of $\rho_{b}$, one has 


$$
u(\rho)=u\left(\rho_{b}\right)+\frac{\mathrm{d} u}{\mathrm{~d} \rho}\left(\rho_{b}\right)\left(\rho-\rho_{b}\right)+\frac{1}{2} \frac{\mathrm{d}^{2} u}{\mathrm{~d} \rho^{2}}(\varsigma)\left(\rho-\rho_{b}\right)^{2}=\frac{1}{2} \frac{\mathrm{d}^{2} u}{\mathrm{~d} \rho^{2}}(\varsigma)\left(\rho-\rho_{b}\right)^{2}
$$

where $\varsigma$ is between $\rho$ and $\rho_{b}$. So the necessary and sufficient convexity condition becomes

$$
\frac{\mathrm{d}^{2} u}{\mathrm{~d} \rho^{2}} \leq 0 \quad \text { in } \quad\left[\frac{1}{2}, 1[\right.
$$

which can be written as

$$
\begin{aligned}
&-f_{2}\left(\rho_{b}\right)=-\left(1-\rho_{b}^{2}\right)^{\frac{1}{2}} g\left(\rho_{b}\right) \leq\left(1-\rho^{2}\right)^{\frac{3}{2}} \frac{\mathrm{d}^{2} f}{\mathrm{~d} \rho^{2}} \\
& \forall \rho \in\left[\frac{1}{2}, 1\right], \quad \forall \rho_{b} \in\left[\frac{1}{2}, 1\right]
\end{aligned}
$$

The function $\frac{\mathrm{d}^{2} f}{\mathrm{~d} \rho^{2}}$ being continuous on $I,(73)$ implies when $\rho$ tends to 1

$$
f_{2}\left(\rho_{b}\right) \geq 0, \quad \forall \rho_{b} \in\left[\frac{1}{2}, 1[\right.
$$

and by the boundary continuity

$$
g\left(\rho_{b}\right) \geq 0 \quad \forall \rho_{b} \in\left[\frac{1}{2}, 1\right] .
$$

The function $g$ being continuous on $I$, by using (73), one also have when $\rho_{b} \rightarrow 1$

$$
\left(1-\rho^{2}\right)^{\frac{3}{2}} \frac{\mathrm{d}^{2} f}{\mathrm{~d} \rho^{2}} \geq 0, \quad \forall \rho \in\left[\frac{1}{2}, 1[\right.
$$

and by the boundary continuity

$$
\frac{\mathrm{d}^{2} f}{\mathrm{~d} \rho^{2}} \geq 0, \quad \forall \rho \in I,
$$

hence (73).

The reverse is direct. Indeed, (73) is obtained by the two inequalities (74) and (76).

- Case $\rho_{b}=1$. Then $x_{b}=1$ and $a\left(x_{b}\right)=0$, and therefore one has

$$
f_{1}\left(\rho_{b}\right)=f(1) \quad \text { and } \quad f_{2}\left(\rho_{b}\right)=0
$$

Then proving $u(\rho) \leq 0$ comes to show that

$$
u(\rho)=\rho f(x(1))-f(x) \leq 0,
$$


i.e.

$$
\rho^{-1} f(x) \geq f(x(1)),
$$

which is always true thanks to (75). In fact the function

$$
\widehat{f}(\rho)=\rho^{-1} f(x)
$$

is decreasing

$$
\frac{\mathrm{d} \widehat{f}}{\mathrm{~d} \rho}=\rho^{-1} \frac{\mathrm{d} f}{\mathrm{~d} \rho}-\rho^{-2} f=-\rho^{-2}\left(f-\rho \frac{\mathrm{d} f}{\mathrm{~d} \rho}\right)=-\rho^{-2} g(\rho) \leq 0 .
$$

So

$$
\widehat{f}(\rho) \geq \widehat{f}(1) \Rightarrow \rho^{-1} f(x) \geq f(x(1)) .
$$

This ends the proof of the proposition.

Proposition 6. Let (22) hold with $g$ non-identically equal to zero. Then $\rho_{\varepsilon}$, as defined by (37), is a continuous bijective application from $I$ to $I$, admitting a first derivative (see (43)) which is continuous and increasing on $\left[\frac{1}{2}, 1[\right.$.

Proof. We examine two cases.

- Case $\rho_{\sigma}=1$. This case is trivial as $\rho_{\varepsilon} \equiv 1$. In fact, from (65), we have

$$
f_{1}(1)>0 \quad \text { and } \quad f_{2}(1)=0 \text {, }
$$

and so

$$
\delta_{f} \equiv \sqrt{\left|f_{1}(1)\right|^{2}+\left|f_{2}(1)\right|^{2}}=f_{1}(1)
$$

which gives

$$
\rho_{\varepsilon}\left(\rho_{\sigma}\right)=\frac{f_{1}(1)}{\delta_{f}}=1
$$

Reciprocally, if $\rho_{\varepsilon}=1$,

$$
\frac{f_{2}\left(\rho_{\sigma}\right)}{\delta_{f}\left(x_{\sigma}\right)}=\left(1-\rho_{\varepsilon}^{2}\right)^{\frac{1}{2}}=0 \Rightarrow f_{2}\left(\rho_{\sigma}\right)=\left(1-\rho_{\sigma}^{2}\right)^{\frac{1}{2}} g\left(\rho_{\sigma}\right)=0,
$$

and so $\rho_{\sigma}=1$. In fact, if $g\left(\rho_{\sigma}\right)=0$ for $\rho_{\sigma} \in\left[\frac{1}{2}, 1[, g\right.$ is decreasing 


$$
0 \leq g(\rho) \leq g\left(\rho_{\sigma}\right)=0 \Rightarrow g(\rho)=0, \quad \forall \rho \in\left[\rho_{\sigma}, 1\right]
$$

which is absurd since $g$ is a function non-identically equal to zero.

- Case $\rho_{\sigma} \neq 1$. In this case $g(\rho)>0$ for all $\rho \in\left[\frac{1}{2}, 1\left[\right.\right.$, and one has $f_{2}(\rho)>0$. Let us derive $\rho_{\varepsilon}=\delta_{f}^{-1} f_{1}$ w.r.t. $\rho$. Set $\delta \equiv \delta_{f}=\sqrt{\left|f_{1}\right|^{2}+\left|f_{2}\right|^{2}}$. One then has

$$
\frac{\mathrm{d} \rho_{\varepsilon}}{\mathrm{d} \rho}=\delta^{-1} \frac{\mathrm{d} f_{1}}{\mathrm{~d} \rho}-\delta^{-2} \frac{\mathrm{d} \delta}{\mathrm{d} \rho} f_{1}=\delta^{-1}\left(\frac{\mathrm{d} f_{1}}{\mathrm{~d} \rho}-\rho_{\varepsilon} \frac{\mathrm{d} \delta}{\mathrm{d} \rho}\right) .
$$

As

$$
\begin{aligned}
\rho_{\varepsilon} \frac{\mathrm{d} \delta}{\mathrm{d} \rho} & =\left(\delta^{-1} f_{1}\right) \delta^{-1}\left(f_{1} \frac{\mathrm{d} f_{1}}{\mathrm{~d} \rho}+f_{2} \frac{\mathrm{d} f_{2}}{\mathrm{~d} \rho}\right)=\delta^{-2} f_{1}^{2} \frac{\mathrm{d} f_{1}}{\mathrm{~d} \rho}+\delta^{-2} f_{1} f_{2} \frac{\mathrm{d} f_{2}}{\mathrm{~d} \rho} \\
& =\frac{\mathrm{d} f_{1}}{\mathrm{~d} \rho}+\delta^{-2} f_{2}\left(f_{1} \frac{\mathrm{d} f_{2}}{\mathrm{~d} \rho}-f_{2} \frac{\mathrm{d} f_{1}}{\mathrm{~d} \rho}\right)
\end{aligned}
$$

so

$$
\frac{\mathrm{d} \rho_{\varepsilon}}{\mathrm{d} \rho}=\delta^{-3} f_{2}\left(f_{2} \frac{\mathrm{d} f_{1}}{\mathrm{~d} \rho}-f_{1} \frac{\mathrm{d} f_{2}}{\mathrm{~d} \rho}\right) .
$$

Then from (24) and (66) we obtain

$$
\frac{\mathrm{d} \rho_{\varepsilon}}{\mathrm{d} \rho}=\delta^{-3} \frac{\mathrm{d} f_{1}}{\mathrm{~d} \rho} f_{2}\left(1-\rho^{2}\right)^{-\frac{1}{2}}\left(\left(1-\rho^{2}\right)^{\frac{1}{2}} f_{2}+f_{1} \rho\right)=\delta^{-3} \frac{\mathrm{d} f_{1}}{\mathrm{~d} \rho} g f,
$$

which is exactly the expression (43). So from (23) and since $f$ et $g$ are strictly positive when $\rho \in\left[\frac{1}{2}, 1\left[\right.\right.$, one concludes that $\rho_{\varepsilon}$ is strictly increasing on $\left[\frac{1}{2}, 1[\right.$ and thus we have a bijection. The smoothness of $\rho_{\varepsilon}$ comes from the smoothness of the composition of these functions.

Proposition 7. Let $\mathcal{F}(\boldsymbol{\sigma})=\overline{\boldsymbol{\sigma}} f\left(y_{\sigma}\right)$ where $f$ is defined by (53). Then $\mathcal{F}$ is convex if and only if the inequalities (54) are verified.

Proof. (54) is a necessary condition because the function arccos (.) exists only if

$$
-1 \leq 1-a(1-y) \leq 1, \quad \forall y \in[-1,1]
$$

Let us show that the condition is also sufficient. In fact, the case where $a=1$ (resp. $a=0)$ is trivial because it corresponds to $f(y)=\cos \left(\frac{1}{3} \arccos (y)\right)=\rho$ (resp. $f(y)=$ $\left.\cos \left(\frac{1}{3} \arccos (1)\right)=1\right)$. We must therefore verify for

$$
0<a<1
$$


One has to note that

$$
w(\rho) \equiv 1-a(1-y)=4 f^{3}-3 f
$$

where $y=4 \rho^{3}-3 \rho$ and $\frac{1}{2}<f \leq 1$. Indeed $f \neq \frac{1}{2}$ because $a(1-y) \neq 2 \Rightarrow w(y) \neq-1$.

By deriving $w$, one obtains

$$
\frac{\mathrm{d} w}{\mathrm{~d} \rho}=3 a\left(4 \rho^{2}-1\right)=3 \frac{\mathrm{d} f}{\mathrm{~d} \rho}\left(4 f^{2}-1\right) \Rightarrow \frac{\mathrm{d} f}{\mathrm{~d} \rho}=a \frac{\left(4 \rho^{2}-1\right)}{\left(4 f^{2}-1\right)} \geq 0
$$

and

$$
\frac{\mathrm{d}^{2} w}{\mathrm{~d} \rho^{2}}=24 a \rho=3\left(\frac{\mathrm{d}^{2} f}{\mathrm{~d} \rho^{2}}\left(4 f^{2}-1\right)+8\left(\frac{\mathrm{d} f}{\mathrm{~d} \rho}\right)^{2} f\right)
$$

Hence following (25), one must verify

$$
\left\{\begin{array}{c}
g(1)=f(y(1))-\frac{\mathrm{d} f}{\mathrm{~d} \rho}(1) \geq 0 \\
a \rho-\left(\frac{\mathrm{d} f}{\mathrm{~d} \rho}\right)^{2} f \geq 0
\end{array} .\right.
$$

The first inequality is direct because $f(y(1))=f(1)=1$ and $\frac{\mathrm{d} f}{\mathrm{~d} \rho}(1)=a$.

Concerning the second one, it is equivalent to

$$
\frac{\mathrm{d} f}{\mathrm{~d} \rho} f^{\frac{1}{2}} \leq a^{\frac{1}{2}} \rho^{\frac{1}{2}} \Leftrightarrow a^{\frac{1}{2}}\left(4 \rho^{2}-1\right) \rho^{-\frac{1}{2}} \leq\left(4 f^{2}-1\right) f^{-\frac{1}{2}} .
$$

We will show that

$$
u(\rho) \leq u(f)
$$

for

$$
u(x) \equiv\left(4 x^{2}-1\right) x^{-\frac{1}{2}}, \quad \forall x \in\left[\frac{1}{2}, 1\right]
$$

In fact, as $u$ is an increasing function

$$
\frac{\mathrm{d} u}{\mathrm{~d} x}=\frac{1}{2} x^{-\frac{3}{2}}\left(1+12 x^{2}\right)>0
$$

and thus it is sufficient to verify

$$
\rho \leq f
$$


i.e.

$$
v(y) \leq v(1-a(1-y)), \quad \forall y \in[-1,1],
$$

for

$$
v(y)=\cos \left(\frac{1}{3} \arccos (y)\right) .
$$

This is always true, because $v(y)$ is an increasing function and

$$
y \leq 1-a(1-y) .
$$

This ends the proof of the proposition.

Proposition 8. The values of $\chi_{\sigma}$ and $\chi_{\epsilon}$ defined by (39) and (46) are opposite, i.e. $\chi_{\sigma}+$ $\chi_{\epsilon}=0$.

Proof. The derivation w.r.t. $\rho_{\sigma}$ of the expression

$$
\cos \chi_{\sigma}=\frac{f\left(x_{\sigma}\right)}{\delta_{f}\left(x_{\sigma}\right)}=\kappa^{2} f\left(x_{\sigma}\right) h\left(x_{\varepsilon}\right),
$$

gives

$$
-\frac{\mathrm{d} \chi_{\sigma}}{\mathrm{d} \rho_{\sigma}} \sin \chi_{\sigma}=\kappa^{2}\left(\frac{\mathrm{d} f}{\mathrm{~d} \rho_{\sigma}} h+f \frac{\mathrm{d} h}{\mathrm{~d} \rho_{\epsilon}} \frac{\mathrm{d} \rho_{\epsilon}}{\mathrm{d} \rho_{\sigma}}\right) .
$$

Let us then multiply by $\frac{\left(1-\rho_{\epsilon}^{2}\right)^{\frac{1}{2}}}{\cos \chi_{\sigma}}$ to obtain

$$
-\frac{\mathrm{d} \chi_{\sigma}}{\mathrm{d} \rho_{\sigma}}\left(1-\rho_{\epsilon}^{2}\right)^{\frac{1}{2}} \tan \chi_{\sigma}=\left(1-\rho_{\epsilon}^{2}\right)^{\frac{1}{2}} \frac{\mathrm{d} f}{\frac{\mathrm{d} \rho_{\sigma}}{f}}+\frac{\left(1-\rho_{\epsilon}^{2}\right)^{\frac{1}{2}} \frac{\mathrm{d} h}{\mathrm{~d} \rho_{\epsilon}}}{h} \frac{\mathrm{d} \rho_{\epsilon}}{\mathrm{d} \rho_{\sigma}} .
$$

But from (18) and (39), one can write

$$
\left\{\begin{array}{l}
\tan \chi_{\sigma}=\frac{a\left(x_{\sigma}\right) f^{\prime}\left(x_{\sigma}\right)}{f\left(x_{\sigma}\right)}=\frac{\left(1-\rho_{\sigma}^{2}\right)^{\frac{1}{2}} \frac{\mathrm{d} f}{\mathrm{~d} \rho_{\sigma}}}{f\left(x_{\sigma}\right)} \\
\tan \chi_{\epsilon}=\frac{a\left(x_{\epsilon}\right) h^{\prime}\left(x_{\epsilon}\right)}{h\left(x_{\epsilon}\right)}=\frac{\left(1-\rho_{\epsilon}^{2}\right)^{\frac{1}{2}} \frac{\mathrm{d} f}{\mathrm{~d} \rho_{\epsilon}}}{h^{\prime}\left(x_{\epsilon}\right)}
\end{array}\right.
$$

and

$$
\left(\left(1-\rho_{\sigma}^{2}\right)^{-\frac{1}{2}}+\frac{\mathrm{d} \chi_{\sigma}}{\mathrm{d} \rho_{\sigma}}\right)\left(1-\rho_{\epsilon}^{2}\right)^{\frac{1}{2}} \tan \chi_{\sigma}+\tan \chi_{\epsilon} \frac{\mathrm{d} \rho_{\epsilon}}{\mathrm{d} \rho_{\sigma}}=0 .
$$


The last term reads (see (42))

$$
\frac{\mathrm{d} \rho_{\epsilon}}{\mathrm{d} \rho_{\sigma}}=\left(\left(1-\rho_{\sigma}^{2}\right)^{-\frac{1}{2}}+\frac{\mathrm{d} \chi_{\sigma}}{\mathrm{d} \rho_{\sigma}}\right)\left(1-\rho_{\varepsilon}^{2}\right)^{\frac{1}{2}}
$$

so that we finally have

$$
\left(\tan \chi_{\sigma}+\tan \chi_{\epsilon}\right) \frac{\mathrm{d} \rho_{\epsilon}}{\mathrm{d} \rho_{\sigma}}=0
$$

i.e.

$$
\tan \chi_{\sigma}+\tan \chi_{\epsilon}=0
$$

Proposition 9. Let $\boldsymbol{\sigma}$ be the solutions of (1). Then, the identity (51) holds.

Proof. Set $\delta_{h} \equiv\left(\left|h\left(x_{\epsilon}\right)\right|^{2}+\left|a\left(x_{\epsilon}\right)\right|^{2}\left|h^{\prime}\left(x_{\epsilon}\right)\right|^{2}\right)^{\frac{1}{2}}$. From (47),

$$
\delta_{h}=h\left(x_{\epsilon}\right)\left(1+\left|a\left(x_{\sigma}\right)\right|^{2}\left|\frac{f^{\prime}\left(x_{\sigma}\right)}{f\left(x_{\sigma}\right)}\right|^{2}\right)^{\frac{1}{2}}=h\left(x_{\epsilon}\right) \frac{\delta_{f}\left(x_{\sigma}\right)}{f\left(x_{\sigma}\right)}=\frac{\kappa^{-2}}{\delta_{f}\left(x_{\sigma}\right)} \frac{\delta_{f}\left(x_{\sigma}\right)}{f\left(x_{\sigma}\right)}=\frac{\kappa^{-2}}{f\left(x_{\sigma}\right)} .
$$

Hence

$$
\begin{aligned}
\mathcal{H}^{\prime}\left(\boldsymbol{\epsilon}_{\sigma}\right) & =\delta_{h}\left(\cos \left(\chi_{\epsilon}\right) \mathbf{n}_{\sigma}+\sin \left(\chi_{\epsilon}\right) \mathbf{n}_{\sigma}^{\perp}\right) \\
& =\frac{\kappa^{-2}}{f\left(x_{\sigma}\right)}\left(\cos \left(\chi_{\epsilon}\right) \mathbf{n}_{\sigma}+\sin \left(\chi_{\epsilon}\right) \mathbf{n}_{\sigma}^{\perp}\right) .
\end{aligned}
$$

Integrating the relation (45) one has also

$$
\mathcal{H}^{\prime}\left(\boldsymbol{\epsilon}_{\sigma}\right)=\frac{\kappa^{-2}}{f\left(x_{\sigma}\right)}\left(\cos \left(\chi_{\sigma}\right) \mathbf{n}_{\sigma}-\sin \left(\chi_{\sigma}\right) \mathbf{n}_{\sigma}^{\perp}\right)
$$

An elementary calculation using (50) shows that

$$
\cos \left(\chi_{\sigma}\right) \mathbf{n}_{\sigma}-\sin \left(\chi_{\sigma}\right) \mathbf{n}_{\sigma}^{\perp}=Q_{1} \mathbf{e}_{1}^{\mathbf{R}_{\sigma}}-Q_{2} \mathbf{e}_{2}^{\mathbf{R}_{\sigma}},
$$

where

$$
\left\{\begin{array}{l}
Q_{1} \equiv \rho_{\epsilon} \cos \left(\chi_{\sigma}\right)-\left(1-\rho_{\epsilon}^{2}\right)^{\frac{1}{2}} \sin \left(\chi_{\sigma}\right) \\
Q_{2} \equiv\left(1-\rho_{\epsilon}^{2}\right)^{\frac{1}{2}} \cos \left(\chi_{\sigma}\right)+\rho_{\epsilon} \sin \left(\chi_{\sigma}\right)
\end{array}\right.
$$


Due to (41)

$$
\left\{\begin{array}{l}
Q_{1}=\cos \left(\theta_{\sigma}-\chi_{\sigma}\right) \cos \left(\chi_{\sigma}\right)-\sin \left(\theta_{\sigma}-\chi_{\sigma}\right) \sin \left(\chi_{\sigma}\right)=\cos \left(\theta_{\sigma}\right)=\rho_{\sigma} \\
Q_{2}=\sin \left(\theta_{\sigma}-\chi_{\sigma}\right) \cos \left(\chi_{\sigma}\right)+\cos \left(\theta_{\sigma}-\chi_{\sigma}\right) \sin \left(\chi_{\sigma}\right)=\sin \left(\theta_{\sigma}\right)=\left(1-\rho_{\sigma}^{2}\right)^{\frac{1}{2}} .
\end{array}\right.
$$

Then, from (4),

$$
\mathcal{H}^{\prime}\left(\boldsymbol{\epsilon}_{\sigma}\right)=\frac{\kappa^{-2}}{f\left(x_{\sigma}\right)}\left(\rho_{\sigma} \mathbf{e}_{1}^{\mathbf{R}_{\sigma}}-\left(1-\rho_{\sigma}^{2}\right)^{\frac{1}{2}} \mathbf{e}_{2}^{\mathbf{R}_{\sigma}}\right)=\frac{\kappa^{-2}}{f\left(x_{\sigma}\right)} \mathbf{N}_{\sigma} .
$$

As $\boldsymbol{\sigma}$ is a solution of (1), then from (8), one finally obtains

$$
\mathcal{H}^{\prime}\left(\boldsymbol{\epsilon}_{\sigma}\right)=\frac{1}{\sigma_{c}} \mathbf{S}_{\sigma}
$$

Proposition 10. Let

$$
\left.f(y)=(1+b y)^{n} ; b \in\right]-1,1[.
$$

If

$$
\left\{\begin{array}{l}
b(n-1) \geq 0 \\
0 \leq b n \leq \frac{1+b}{9}
\end{array}\right.
$$

then $\mathcal{F}(\boldsymbol{\sigma})=\overline{\boldsymbol{\sigma}} f\left(y_{\sigma}\right)$ is convex.

Proof. Let us verify the inequalities (29). Both are direct due to the assumptions (80). Indeed we have the first inequality

$$
g(1)=(1+b-9 n b)(1+b y)^{n-1} \geq 0 .
$$

The second one is also trivial because of

$$
f^{\prime}(y)=n b(1+b y)^{n-1} \geq 0
$$

and

$$
f^{\prime \prime}(y)=n(n-1) b^{2}(1+b y)^{n-2} \geq 0 .
$$




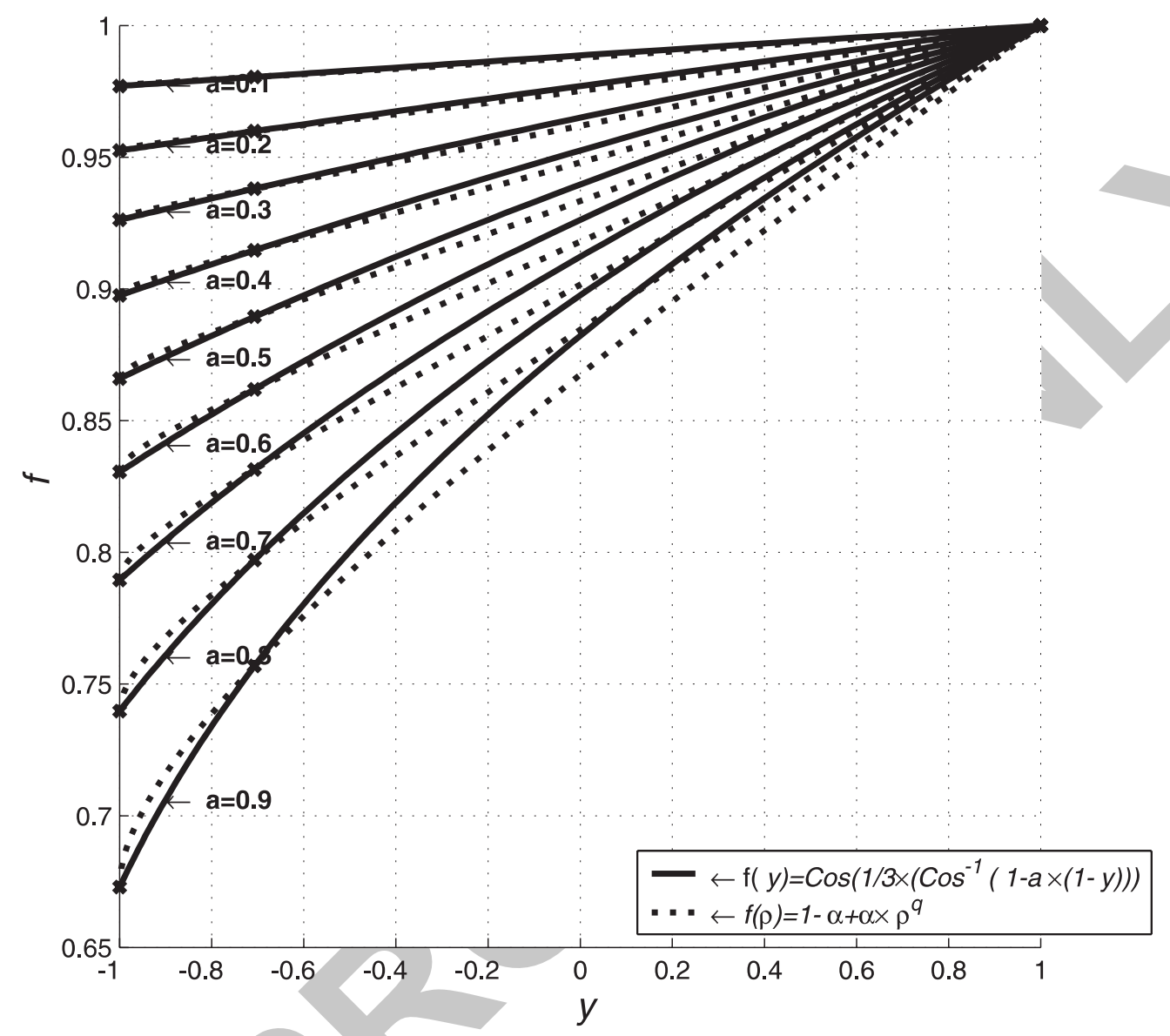

Figure 12. For fixed $a$ value comparison between "cosinus" and "power law" shape function $f$ versus $y$.

\section{APPENDIX A}

First we will examine the convexity conditions of a "power law" shape function $f(\rho)$. Then this function is compared in the Figure 12 with the "cosinus" formulation of $f(y)$, see (53).

Proposition 11. Let $\mathcal{F}(\boldsymbol{\sigma})=\overline{\boldsymbol{\sigma}} f\left(\rho_{\sigma}\right)$ where $f$ is defined by

$$
f(\rho)=1-\alpha+\alpha \rho^{q},
$$

where the parameters $\alpha$ and $q$ are such that

$$
\begin{cases}0 \leq \alpha q \leq 1 & \text { if } \quad q \geq 1 \\ \alpha q \leq 0 & \text { otherwise. }\end{cases}
$$

Then $\mathcal{F}$ is convex if and only if the inequalities (82) are verified. 
Proof. The condition of the strict positivity of $f$ and also the convexity condition (25) imply in a reciprocal manner

$$
\begin{cases}f(\rho) & >0 \\ \alpha q & \leq 1 \\ \alpha q(1-q) \leq 0\end{cases}
$$

for all $\rho \in I$. Two cases are investigated:

- Case $q \geq 1$. The condition (83) is equivalent to

$$
\left\{\begin{array}{r}
f(\rho)>0 \\
0 \leq \alpha q \leq 1
\end{array}\right.
$$

The first inequality est redundant because in this case $f$ is increasing $\left(\frac{\mathrm{d} f}{\mathrm{~d} \rho}=\alpha q \rho^{q-1} \geq 0\right)$, and hence

$$
f(\rho) \geq f\left(2^{-1}\right) \geq 1-\alpha\left(1-2^{-q}\right)>1-\alpha q \geq 0 .
$$

Therefore it suffices to verify

$$
0 \leq \alpha q \leq 1
$$

- Case $q<1$. The condition (83) becomes

$$
\left\{\begin{array}{l}
f(\rho)>0 \\
\alpha q \leq 0
\end{array}\right.
$$

As $f$ is decreasing, we have

$$
f(\rho) \geq f(1)=1
$$

then (85) is equivalent to

$$
\alpha q \leq 0
$$

Figure 12 illustrates, for values of $a=\frac{1}{10}, \frac{2}{10}, \ldots, \frac{9}{10}$, the comparison with the function

$$
f(y)=\cos \left(\frac{1}{3} \arccos (1-a(1-y))\right) \text {. }
$$


The couples $(\alpha, q)$ are chosen such that the two curves coincide at points $y=-1,-\frac{\sqrt{2}}{2}$, +1 (resp. $\left.\rho=2^{-1}, 2^{-\frac{1}{2}}, 1\right)$, i.e.

$$
\left\{\begin{array}{l}
q=\frac{2}{\ln (2)} \ln \left(\frac{1-f\left(-\frac{\sqrt{2}}{2}\right)}{f\left(-\frac{\sqrt{2}}{2}\right)-f(-1)}\right) \\
\alpha=\frac{f\left(-\frac{\sqrt{2}}{2}\right)-1}{2^{-\frac{q}{2}}-1}
\end{array}\right.
$$

where

$$
\left\{\begin{array}{l}
f(-1)=\cos \left(\frac{1}{3} \arccos (1-2 a)\right) \\
f\left(-\frac{\sqrt{2}}{2}\right)=\cos \left(\frac{1}{3} \arccos \left(1-a\left(1+\frac{\sqrt{2}}{2}\right)\right)\right) .
\end{array}\right.
$$

Figure 13 gives the couples $(\alpha, q)$ as a function of $a$. Note that the convexity condition (82) is clearly verified.

\section{APPENDIX B}

We are going to give here a necessary and sufficient convexity condition for the shape function introduced by Raniecki and Mroz [3].

Proposition 12. Let $\mathcal{F}(\boldsymbol{\sigma})=\overline{\boldsymbol{\sigma}} f\left(y_{\sigma}\right)$ where $f$ is defined by

$$
f(y)=1+\alpha(1-\exp (-\beta(1+y))) ; \beta>0 \text { and } \alpha \neq 0
$$

Then $\mathcal{F}$ is convex if and only if

$$
\left\{\begin{aligned}
0<\alpha & \leq b(\beta) \equiv \frac{1}{\exp (-2 \beta)(1+9 \beta)-1} \\
\beta & \leq \frac{8}{27}
\end{aligned}\right.
$$

Figure 14 shows the evolution of $b$ as a function of $\beta$.

Proof. A simple calculation starting from (68) shows that

$$
\frac{\mathrm{d}^{2} f}{\mathrm{~d} \rho^{2}}=6 \alpha \beta \exp (-\beta(1+y)) u(\rho),
$$




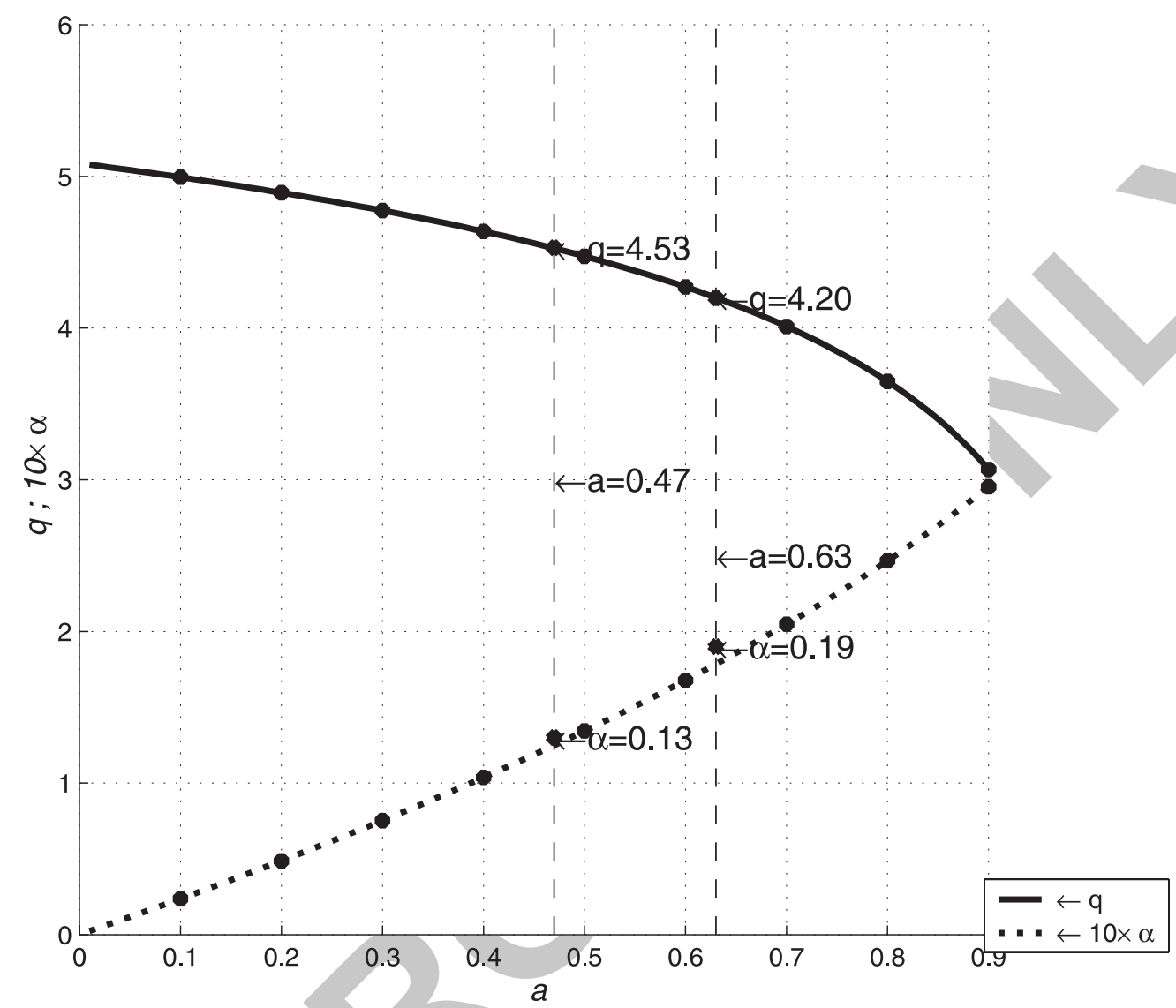

Figure 13. Determination of the couples $(\alpha, q)$ as $a$ function.

with

$$
u(\rho)=4 \rho-\frac{3}{2}\left(4 \rho^{2}-1\right)^{2} \beta
$$

Then

- for $\alpha<0$

$$
\frac{\mathrm{d}^{2} f}{\mathrm{~d} \rho^{2}} \ngtr 0, \quad \forall \rho \in I
$$

because

$$
u\left(\frac{1}{2}\right)=2 \geq 0
$$




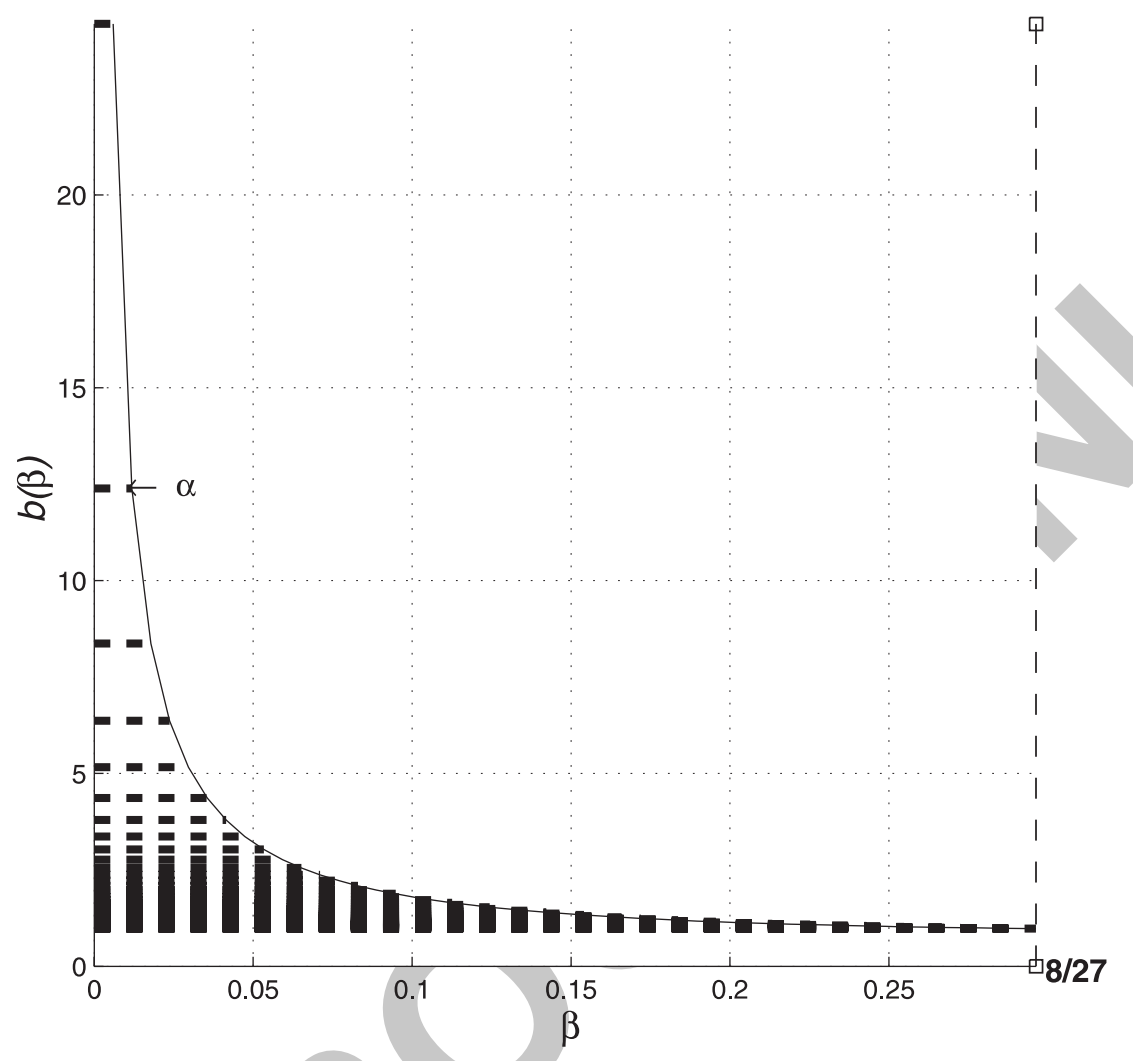

Figure 14. Evolution of $b$ versus $\beta$ ( $b$ and $\beta$ full filled the convexity conditions of the yield surface in the stress space).

- for $\alpha>0$, the inequality

$$
\frac{\mathrm{d}^{2} f}{\mathrm{~d} \rho^{2}} \geq 0, \quad \forall \rho \in I
$$

is equivalent to

$$
u(\rho) \geq 0, \quad \forall \rho \in I .
$$

We already have $u\left(\frac{1}{2}\right) \geq 0$. The case to examine is $\rho \neq \frac{1}{2}$ which is equivalent to

$$
\left.\left.\beta \leq v(\rho) \equiv \frac{8 \rho}{3\left(4 \rho^{2}-1\right)^{2}}, \quad \forall \rho \in\right] \frac{1}{2}, 1\right] .
$$

As $v$ is a decreasing function on $\left.] \frac{1}{2}, 1\right]$, because 


$$
\frac{\mathrm{d} v}{\mathrm{~d} \rho}=-\frac{8}{3}\left(4 \rho^{2}-1\right)^{-3}\left(1+12 \rho^{2}\right)<0
$$

so (91) is equivalent to

$$
\beta \leq v(1)=\frac{8}{27}
$$

Finally, following (25), it is neccesary and sufficient that (see (67))

$$
g(1)=f(1)-9 f^{\prime}(1)=1+\alpha(1-\exp (-2 \beta)(1+9 \beta)) \geq 0
$$

i.e.

$$
\frac{1}{\alpha} \geq w(\beta) \equiv \exp (-2 \beta)(1+9 \beta)-1
$$

But for $\beta \leq \frac{8}{27}$

$$
\frac{\mathrm{d} w}{\mathrm{~d} \beta}=(7-18 \beta) \exp (-2 \beta) \geq \frac{5}{3} \exp (-2 \beta)>0,
$$

thus for a parameter $\varsigma$ between 0 and $\beta$,

$$
\beta w(\beta)=w(0)+\frac{\mathrm{d} w}{\mathrm{~d} \beta}(\varsigma) \beta=\frac{\mathrm{d} w}{\mathrm{~d} \beta}(\varsigma) \beta>0 .
$$

\section{REFERENCES}

[1] Burkart, M. W. and Read, T. A. Transactions of the TMS-AIME, 197, 1516-1524 (1953).

[2] Raniecki, B. and Lexcellent, C. Thermodynamics of isotropic pseudoelasticity in shape memory alloys. European Journal of Mechanics A, 17, 185-205 (1998).

[3] Raniecki, B. and Mroz, S. Yield or martensitic phase transformation conditions and dissipation functions for isotropic, pressure-insensitive alloys exhibiting SD effect. Acta Mechanica, 195, 81-102 (2008).

[4] Podgorski, J. General failure criterion for isotropic media. Journal of Engineering and Mechanics (ASCE), 111, 188-199 (1985).

[5] Podgorski, J. Limit state condition and the dissipation function for isotropic materials. Archives of Mechanics, 36, 323-342 (1984).

[6] Lode, W. Versuche fiber den EinluB der mittlerea Hauptspannung auf das FlieBen. der Metalle Eisen, Kupfer und Nickel. Zeitschrift für Angewandte Mathematik und Physik, 86, 913-939 (1926).

[7] Bigoni, D. and Piccolroaz, A. Yield criteria for quasibrittle and functional materials. International Journal of Solids and Structures, 41, 2855-2878 (2004).

[8] Sadjadpour, A. and Bhattacharya, K. A micromechanics inspired model for shape-memory alloy. Smart Material Systems and Structures, to appear.

[9] Bouvet, C., Calloch, S. and Lexcellent, C. Mechanical behavior of a Cu-Al-Be shape memory alloy under multiaxial proportional and non-proportional loadings. Journal of Engineering Material and Technology, 124, 112-124 (2002).

[10] Laverhne-Taillard, K., Calloch, S., Arbab Chirani, S. and Lexcellent, C. Multiaxial shape memory effect and superelasticity. Strain, to appear. 